Relativistic configuration interaction calculations of energy levels, isotope shifts, hyperfine structures, and transition rates in the $2 s^{2} 2 p^{2}-2 s 2 p^{3}$ transition array for the carbon-like sequence

This article has been downloaded from IOPscience. Please scroll down to see the full text article.

2010 J. Phys. B: At. Mol. Opt. Phys. 43074023

(http://iopscience.iop.org/0953-4075/43/7/074023)

View the table of contents for this issue, or go to the journal homepage for more

Download details:

IP Address: 130.235.64.24

The article was downloaded on 17/07/2010 at 12:31

Please note that terms and conditions apply. 


\title{
Relativistic configuration interaction calculations of energy levels, isotope shifts, hyperfine structures, and transition rates in the $2 s^{2} 2 p^{2}-2 s 2 p^{3}$ transition array for the carbon-like sequence
}

\author{
Per Jönsson $^{1}$ and Jacek Bieroń ${ }^{2}$ \\ ${ }^{1}$ Nature, Environment, Society, Malmö University, S-20506, Malmö, Sweden \\ ${ }^{2}$ Instytut Fizyki imienia Mariana Smoluchowskiego, Uniwersytet Jagielloński, Reymonta 4, \\ 30-059 Kraków, Poland
}

Received 11 August 2009, in final form 12 October 2009

Published 19 March 2010

Online at stacks.iop.org/JPhysB/43/074023

\begin{abstract}
Energy levels, fine-structure separations, specific mass shift parameters, isotope shifts, hyperfine interaction constants, Landé $g_{J}$-factors and transition probabilities are reported for the $2 s^{2} 2 p^{2}-2 s 2 p^{3}$ transition array in N II, O III, F IV, Ne V and Ti XVII. Wavefunctions were determined using the multiconfiguration Dirac-Hartree-Fock method with account for valence, core-valence and core-core correlation effects. The transition energies and rates are compared with experimental data and with values from other calculations.
\end{abstract}

\section{Introduction}

Light atoms are important in astrophysical applications and transition data can be used in the determination of element abundances and plasma diagnostics [1-3]. A number of general computer codes such as SUPERSTRUCTURE, CIV3 and ATSP2K have been developed [4-6] to provide for these extensive data needs. These codes rely on the Breit-Pauli approximation [7, 8], in which the atomic state function $\Psi\left(\gamma J M_{J}\right)$ is a linear combination of configuration state functions (CSF) of the form

$$
\Psi\left(\gamma J M_{J}\right)=\sum_{L S} \sum_{j} c_{j}(L S) \Phi\left(\gamma_{j} L S J M_{J}\right)
$$

where $\gamma$ usually represents the dominant configuration and any additional quantum numbers required for uniquely specifying the state. The configuration state functions $\Phi\left(\gamma_{j} L S J M_{J}\right)$ are built from a basis of one-electron spin-orbitals. The expansion coefficients $c_{j}(L S)$ and the corresponding energy $E(\gamma J)$ are an eigenvector and eigenvalue, respectively, of the interaction matrix of these CSFs as defined by the BreitPauli Hamiltonian. In accurate calculations the Hamiltonian includes the mass correction, the one- and two-body Darwin terms, the spin-spin contact term, and the $J$-dependent spinorbit, spin-other-orbit and spin-spin interactions. For massive calculations, where computational resources might be a problem, the operators are often limited to one-body mass correction, the Darwin term and the spin-orbit term [9].

For light elements the sum (1) is dominated by one $L S$ term and the rate for an intercombination transition depends critically on the admixture of CSFs with different $L S$ designations. A perturbation analysis shows that mixing of different $L S$ terms is affected by term energy separations. Though it is possible, in simple cases, to correct a computed transition rate for such an error, it is simpler to first adjust the $L S$ term energies in a Breit-Pauli calculation so that, for selected $J$ values, the separation of terms is in close agreement with observed energies. Such a procedure is called fine-tuning. When judiciously applied, it has been shown to yield consistent sets of transition data even in cases where values from different uncorrected calculations are quite far away [10].

Although highly competitive for light atoms, especially when fine-tuning is applied, the Breit-Pauli approximation has some drawbacks. Relativistic effects manifest themselves qualitatively as orbital contractions (a direct relativistic effect), so that the inner electrons move in orbits closer to the 
Table 1. Computed energies for F IV as functions of the increasing active sets, where $n=3$ denotes the orbital set with maximal principal quantum number $n=3$, etc. MR denotes calculations using the $n=8$ active set, but with an enlarged multireference set. The experimental energies are those of [40].

\begin{tabular}{llrrrrrrrrr}
\hline Term & $J$ & $n=3$ & $n=4$ & $n=5$ & $n=6$ & $n=7$ & $n=8$ & MR & QED & Exp \\
\hline $2 \mathrm{~s}^{2} 2 \mathrm{p}^{2}{ }^{3} \mathrm{P}$ & 0 & 0 & 0 & 0 & 0 & 0 & 0 & & 0 & 0 \\
& 1 & 222.7 & 240.4 & 242.7 & 244.3 & 244.7 & 245.0 & 226.4 & 226.9 & 226.0 \\
& 2 & 608.7 & 615.1 & 616.1 & 615.1 & 615.1 & 615.0 & 611.6 & 613.2 & 614.0 \\
$2 \mathrm{~s}^{2} 2 \mathrm{p}^{21} \mathrm{D}$ & 2 & 27370.2 & 25961.9 & 25596.4 & 25427.1 & 25411.2 & 25395.5 & 25367.8 & 25369.0 & 25238.2 \\
$2 \mathrm{~s}^{2} 2 \mathrm{p}^{2}{ }^{1} \mathrm{~S}$ & 0 & 56739.8 & 54472.1 & 53885.7 & 53653.6 & 53633.0 & 53616.0 & 53746.2 & 53744.8 & 53541.2 \\
$2 \mathrm{~s} 2 \mathrm{p}^{3}{ }^{5} \mathrm{~S}^{o}$ & 2 & 70534.1 & 72279.0 & 72947.6 & 73459.1 & 73682.7 & 73792.0 & 73949.4 & 73898.2 & 74194.7 \\
$2 \mathrm{~s} 2 \mathrm{p}^{3}{ }^{3} \mathrm{D}^{o}$ & 3 & 149981.6 & 148424.7 & 148178.4 & 148149.8 & 148142.0 & 148131.6 & 147964.2 & 147911.2 & 147843.0 \\
& 2 & 150008.7 & 148434.0 & 148183.8 & 148151.7 & 148142.3 & 148132.6 & 148011.0 & 147958.0 & 147888.7 \\
& 1 & 150023.3 & 148447.5 & 148197.1 & 148164.6 & 148155.0 & 148144.1 & 148023.7 & 147970.7 & 147903.5 \\
$2 \mathrm{~s} 2 \mathrm{p}^{3}{ }^{3} \mathrm{P}^{o}$ & 2 & 179445.7 & 176653.3 & 176004.6 & 175710.6 & 175590.3 & 175559.6 & 175509.8 & 175457.7 & 175236.8 \\
& 1 & 179446.6 & 176655.6 & 176006.0 & 175712.8 & 175592.6 & 175562.4 & 175513.7 & 174461.6 & 175241.9 \\
& 0 & 179469.7 & 176689.6 & 176043.6 & 175757.5 & 175639.6 & 175610.7 & 175540.6 & 175488.5 & 175263.9 \\
$2 \mathrm{~s} 2 \mathrm{p}^{3}{ }^{1} \mathrm{D}^{o}$ & 2 & 234577.1 & 230902.7 & 230115.2 & 229863.4 & 229764.2 & 229693.6 & 229263.2 & 229205.2 & 228903.8 \\
$2 \mathrm{~s} 2 \mathrm{p}^{3}{ }^{3} \mathrm{~S}^{o}$ & 1 & 241919.3 & 239765.5 & 239130.3 & 238958.1 & 238839.5 & 238825.0 & 238564.0 & 238502.1 & 238296.7 \\
$2 \mathrm{~s} 2 \mathrm{p}^{3} \mathrm{P}^{o}$ & 1 & 265416.8 & 260294.5 & 259085.7 & 258565.7 & 258407.6 & 258318.9 & 257929.5 & 257873.0 & 257386.5 \\
\hline
\end{tabular}

nucleus. The amount of the contraction depends critically on the orbital symmetry - the largest contraction affects orbitals with the $s$ angular symmetry. Orbital contraction has an important secondary, indirect relativistic effect on the more weakly bound high-angular-symmetry electrons [11], which undergo radial expansion and become, qualitatively, still less weakly bound. In fully relativistic methods based on the Dirac-Coulomb Hamiltonian these effects are described directly, through the shape of the radial orbitals. When methods based on the Breit-Pauli approximation are used, the relativistic rearrangement of the electrons is described in a less efficient way through the configuration expansion coefficients. The direct and indirect relativistic effects grow rapidly with increasing nuclear charge, and eventually the nonrelativistic orbital bases used in the Breit-Pauli calculations become inadequate in describing the system.

As alternatives to the general purpose codes in the Breit-Pauli approximation, fully relativistic codes, such as GRASP2K [12] and MCDFGME [13], can be used. Both are based on the multiconfiguration Dirac-Hartree-Fock method and can be applied to general systems with several open shells. In the present paper the GRASP2K package has been used. It implements a biorthogonal transformation technique that permits initial and final states in a transition array to be optimized separately [14], which, in many cases, leads to more accurate values of the calculated rates. The package also contains modules for computing diagonal and off-diagonal hyperfine interaction constants [15], isotope shifts [16], as well as Landé $g_{J}$-factors and splittings of magnetic substates in intermediate and strong magnetic fields [17].

The purpose of the present paper is threefold. Firstly, we provide data for plasma diagnosis and line profile modelling. We used the GRASP2K code to compute energy levels, fine-structure separations, specific mass shift parameters, hyperfine interaction constants, Landé $g_{J}$-factors and transition probabilities for the $2 \mathrm{~s}^{2} 2 \mathrm{p}^{2}-2 \mathrm{~s} 2 \mathrm{p}^{3}$ transition array in N II, O III, F IV, Ne V and Ti XVII. Nitrogen and oxygen are abundant elements in astrophysical objects and emission lines of carbon-like ions are useful in the diagnosis of the solar, astrophysical and fusion plasmas [18]. There are very little data on hyperfine structure and isotope shifts available in the literature and computed parameters should support detailed modelling of the line profiles in high-resolution spectra [19]. The second purpose of the present paper is to test the capability of the GRASP2K code to produce large volumes of spectroscopic data with high accuracy necessary in astrophysical, plasma, spectroscopy, as well as other modern applications. In addition, in this paper transition data for N II, O III, F IV and Ne V are critically evaluated and compared with available experimental values and with the results from recent Breit-Pauli calculations [20-24], with the aim to establish an optimal combination of semi-relativistic Breit-Pauli and fully relativistic Dirac-Hartree-Fock methods in application to atomic spectroscopy ab initio calculations.

\section{Computational procedure}

The multiconfiguration Dirac-Hartree-Fock method is described in detail in a recent monograph by Grant [27] and here we only give a brief outline. Starting from the DiracCoulomb Hamiltonian

$$
H_{\mathrm{DC}}=\sum_{i=1}^{N}\left[c \boldsymbol{\alpha}_{i} \cdot \boldsymbol{p}_{i}+\left(\beta_{i}-1\right) c^{2}+V_{i}^{N}\right]+\sum_{i<j}^{N} 1 / r_{i j},
$$

where $V^{N}$ is the monopole part of the electron-nucleus Coulomb interaction, the atomic state functions describing different fine structure levels are obtained as linear combinations of symmetry adapted configuration state functions (CSFs)

$$
\Psi\left(\gamma J M_{J}\right)=\sum_{j} c_{j} \Phi\left(\gamma_{j} J M_{J}\right) .
$$

Here $J$ and $M_{J}$ are the angular quantum numbers, while $\gamma_{j}$ denotes other appropriate labelling of the configuration state functions, for example parity, orbital occupancy and coupling scheme. The configuration state functions $\Phi\left(\gamma_{j} J M_{J}\right)$ are built from products of one-electron Dirac orbitals. In the relativistic self-consistent field procedure both the radial parts of the Dirac orbitals and the expansion coefficients 
Table 2. Energies for N II, O III and F IV from multireference RCI calculations with QED corrections. The energies are given in units of $\mathrm{cm}^{-1}$ relative to a ground state energy of zero. Splitting of energy levels relative to the lowest level for the term. The experimental energies are those of [40].

\begin{tabular}{|c|c|c|c|c|c|c|c|}
\hline \multirow[b]{2}{*}{ Level } & \multirow[b]{2}{*}{$J$} & \multicolumn{3}{|c|}{ Level $\left(\mathrm{cm}^{-1}\right)$} & \multicolumn{3}{|c|}{ Splitting $\left(\mathrm{cm}^{-1}\right)$} \\
\hline & & Theory & Observation & Difference & Theory & Observation & Difference \\
\hline & & & & & & & \\
\hline \multirow[t]{3}{*}{$2 \mathrm{~s}^{2} 2 \mathrm{p}^{2}{ }^{3} \mathrm{P}$} & $\overline{0}$ & 0 & 0 & 0 & & & \\
\hline & 1 & 49.3 & 48.7 & 0.6 & 49.3 & 48.7 & 0.6 \\
\hline & 2 & 129.2 & 130.8 & -1.6 & 129.2 & 130.8 & -1.6 \\
\hline $2 s^{2} 2 p^{21} D$ & 2 & 15458.8 & 15316.2 & 142.6 & & & \\
\hline $2 s^{2} 2 p^{21} s$ & 0 & 32938.1 & 32688.8 & 249.3 & & & \\
\hline $2 \mathrm{~s} 2 \mathrm{p}^{35} \mathrm{~S}^{o}$ & 2 & 46480.2 & 46784.6 & -304.4 & & & \\
\hline \multirow[t]{3}{*}{$2 \mathrm{~s} 2 \mathrm{p}^{33} \mathrm{D}^{o}$} & 3 & 92356.0 & 92237.2 & 118.8 & & & \\
\hline & 2 & 92367.9 & 92250.3 & 117.6 & 11.9 & 13.1 & -1.2 \\
\hline & 1 & 92369.0 & 92251.8 & 117.2 & 13.0 & 14.6 & -1.6 \\
\hline \multirow{3}{*}{$2 \mathrm{~s} 2 \mathrm{p}^{3}{ }^{3} \mathrm{P}^{o}$} & 2 & 109693.1 & 109217.6 & 475.5 & & & \\
\hline & 1 & 109693.2 & 109216.6 & 476.6 & 0.1 & -1.1 & 1.2 \\
\hline & 0 & 109709.4 & 109223.5 & 485.9 & 16.3 & 5.9 & 10.4 \\
\hline \multirow[t]{2}{*}{$2 \mathrm{~s} 2 \mathrm{p}^{3}{ }^{1} \mathrm{D}^{o}$} & 2 & 144705.9 & 144187.9 & 518.0 & & & \\
\hline & \multicolumn{7}{|c|}{ O III } \\
\hline \multirow[t]{3}{*}{$2 \mathrm{~s}^{2} 2 \mathrm{p}^{23} \mathrm{P}$} & $\overline{0}$ & 0 & 0 & 0 & & & \\
\hline & 1 & 113.6 & 113.2 & 0.4 & 113.6 & 113.2 & 0.4 \\
\hline & 2 & 305.0 & 306.2 & -1.2 & 305.2 & 306.2 & -1.0 \\
\hline $2 \mathrm{~s}^{2} 2 \mathrm{p}^{21} \mathrm{D}$ & 2 & 20400.4 & 20273.3 & 127.1 & & & \\
\hline $2 s^{2} 2 p^{2}{ }^{1} s$ & 0 & 43393.4 & 43185.7 & 207.7 & & & \\
\hline $2 \mathrm{~s} 2 \mathrm{p}^{35} \mathrm{~S}^{o}$ & 2 & 60022.8 & 60324.8 & -302.0 & & & \\
\hline \multirow[t]{3}{*}{$2 \mathrm{~s} 2 \mathrm{p}^{3}{ }^{3} \mathrm{D}^{o}$} & 3 & 120098.9 & 120025.2 & 73.7 & & & \\
\hline & 2 & 120125.8 & 120053.4 & 72.4 & 26.9 & 28.2 & -1.3 \\
\hline & 1 & 120131.4 & 120058.2 & 73.2 & 32.5 & 33.0 & -0.5 \\
\hline \multirow[t]{3}{*}{$2 \mathrm{~s} 2 \mathrm{p}^{3}{ }^{3} \mathrm{P}^{o}$} & 2 & 142647.7 & 142381.0 & 266.7 & & & \\
\hline & 1 & 142649.6 & 142381.8 & 267.8 & 1.9 & 0.8 & 1.1 \\
\hline & 0 & 142669.7 & 142393.5 & 276.2 & 22.0 & 12.5 & 9.5 \\
\hline $2 \mathrm{~s} 2 \mathrm{p}^{31} \mathrm{D}^{o}$ & 2 & 187366.5 & 187054.0 & 312.5 & & & \\
\hline $2 \mathrm{~s} 2 \mathrm{p}^{3}{ }^{3} \mathrm{~S}^{o}$ & 1 & 197299.5 & 197087.7 & 211.8 & & & \\
\hline \multirow[t]{2}{*}{$2 \mathrm{~s} 2 \mathrm{p}^{3}{ }^{1} \mathrm{P}^{o}$} & 1 & 211171.1 & 210461.8 & 709.3 & & & \\
\hline & \multicolumn{7}{|c|}{ F IV } \\
\hline \multirow{3}{*}{$2 \mathrm{~s}^{2} 2 \mathrm{p}^{2} \mathrm{P}$} & $\overline{0}$ & 0 & 0 & 0 & & & \\
\hline & 1 & 226.9 & 226.0 & 0.9 & 226.9 & 226.0 & 0.9 \\
\hline & 2 & 613.2 & 614.0 & -0.8 & 613.2 & 614.0 & -0.8 \\
\hline $2 \mathrm{~s}^{2} 2 \mathrm{p}^{21} \mathrm{D}$ & 2 & 25369.0 & 25238.2 & 130.8 & & & \\
\hline $2 s^{2} 2 p^{21} S$ & 0 & 53744.8 & 53541.2 & 203.6 & & & \\
\hline $2 \mathrm{~s} 2 \mathrm{p}^{35} \mathrm{~S}^{o}$ & 2 & 73898.2 & 74194.7 & -296.5 & & & \\
\hline \multirow[t]{3}{*}{$2 \mathrm{~s} 2 \mathrm{p}^{3}{ }^{3} \mathrm{D}^{o}$} & 3 & 147911.2 & 147843.0 & 68.2 & & & \\
\hline & 2 & 147958.0 & 147888.7 & 69.3 & 46.8 & 45.7 & 1.1 \\
\hline & 1 & 147970.7 & 147903.5 & 67.2 & 59.5 & 60.5 & -1.0 \\
\hline \multirow[t]{3}{*}{$2 \mathrm{~s} 2 \mathrm{p}^{3}{ }^{3} \mathrm{P}^{o}$} & 2 & 175457.7 & 175236.8 & 220.9 & & & \\
\hline & 1 & 175461.6 & 175241.9 & 219.7 & 3.9 & 5.1 & -1.2 \\
\hline & 0 & 175488.5 & 175263.9 & 224.6 & 30.8 & 27.1 & 3.7 \\
\hline $2 \mathrm{~s} 2 \mathrm{p}^{3}{ }^{3} \mathrm{~S}^{o}$ & 1 & 229205.2 & 228903.8 & 301.4 & & & \\
\hline $2 \mathrm{~s} 2 \mathrm{p}^{31} \mathrm{D}^{o}$ & 2 & 238502.1 & 238296.7 & 205.4 & & & \\
\hline $2 \mathrm{~s} 2 \mathrm{p}^{3}{ }^{1} \mathrm{P}^{o}$ & 1 & 257873.0 & 257386.5 & 486.5 & & & \\
\hline
\end{tabular}

are optimized to self-consistency. In subsequent relativistic configuration interaction (RCI) calculations the transverse photon interaction

$$
\begin{aligned}
H_{\text {Breit }} & =-\sum_{i<j}^{N}\left[\boldsymbol{\alpha}_{i} \cdot \boldsymbol{\alpha}_{j} \frac{\cos \left(\omega_{i j} r_{i j} / c\right)}{r_{i j}}\right. \\
+ & \left.\left(\boldsymbol{\alpha}_{i} \cdot \nabla_{i}\right)\left(\boldsymbol{\alpha}_{i} \cdot \nabla_{i}\right) \frac{\cos \left(\omega_{i j} r_{i j} / c\right)-1}{\omega_{i j}^{2} r_{i j} / c^{2}}\right]
\end{aligned}
$$

may be included in the Hamiltonian [28]. The photon frequency $\omega_{i j}$ used by GRASP2K in calculating the matrix elements of the transverse photon interaction is taken as the difference of the diagonal Lagrange multipliers $\epsilon_{i}$ and $\epsilon_{j}$ associated with Dirac orbitals. In general, diagonal Lagrange multipliers are approximate electron removal energies only when orbitals are spectroscopic and singly occupied. Thus, (4) it is not known how well the code can determine the full transverse photon interaction when correlation orbitals are 
Table 3. Energies for Ne V and Ti XVII from multireference RCI calculations with QED corrections. The energies are given in units of $\mathrm{cm}^{-1}$ relative to a ground state energy of zero. Splitting of energy levels relative to the lowest level for the term. The experimental energies are those of [40].

\begin{tabular}{|c|c|c|c|c|c|c|c|}
\hline \multirow[b]{2}{*}{ Level } & \multirow[b]{2}{*}{$J$} & \multicolumn{3}{|c|}{ Level $\left(\mathrm{cm}^{-1}\right)$} & \multicolumn{3}{|c|}{ Splitting $\left(\mathrm{cm}^{-1}\right)$} \\
\hline & & Theory & Observation & Difference & Theory & Observation & Difference \\
\hline & & & & & & & \\
\hline \multirow[t]{3}{*}{$2 \mathrm{~s}^{2} 2 \mathrm{p}^{2}{ }^{3} \mathrm{P}$} & $\overline{0}$ & 0 & 0 & 0 & & & \\
\hline & 1 & 411.3 & 411.2 & 0.1 & 411.3 & 411.2 & 0.1 \\
\hline & 2 & 1108.4 & 1109.5 & -1.1 & 1108.4 & 1109.5 & -1.1 \\
\hline $2 s^{2} 2 p^{21} D$ & 2 & 30289.9 & 30290.7 & -0.8 & & & \\
\hline $2 s^{2} 2 p^{2}{ }^{1} s$ & 0 & 63986.2 & 63915.4 & 70.8 & & & \\
\hline $2 \mathrm{~s} 2 \mathrm{p}^{35} \mathrm{~S}^{o}$ & 2 & 87965.1 & 88399.5 & -434.4 & & & \\
\hline \multirow[t]{3}{*}{$2 \mathrm{~s} 2 \mathrm{p}^{3}{ }^{3} \mathrm{D}^{o}$} & 3 & 175779.2 & 175832.3 & -53.1 & & & \\
\hline & 2 & 175849.4 & 175902.7 & -53.3 & 70.2 & 70.4 & -0.2 \\
\hline & 1 & 175873.0 & 175925.0 & -52.0 & 93.8 & 92.7 & 1.1 \\
\hline \multirow{3}{*}{$2 \mathrm{~s} 2 \mathrm{p}^{3}{ }^{3} \mathrm{P}^{o}$} & 2 & 208217.6 & 208151.3 & 66.3 & & & \\
\hline & 1 & 208222.1 & 208153.3 & 68.8 & 4.5 & 2.0 & 2.5 \\
\hline & 0 & 208258.7 & 208185 & 73.7 & 41.1 & 33.7 & 7.4 \\
\hline $2 \mathrm{~s} 2 \mathrm{p}^{3}{ }^{3} \mathrm{~S}^{o}$ & 1 & 270715.1 & 270552.9 & 162.2 & & & \\
\hline $2 \mathrm{~s} 2 \mathrm{p}^{31} \mathrm{D}^{o}$ & 2 & 279434.6 & 279371.2 & 63.4 & & & \\
\hline \multirow[t]{2}{*}{$2 \mathrm{~s} 2 \mathrm{p}^{3}{ }^{1} \mathrm{P}^{o}$} & 1 & 304127.1 & 303819.2 & 307.9 & & & \\
\hline & \multicolumn{7}{|c|}{ Ti XVII } \\
\hline \multirow[t]{3}{*}{$2 \mathrm{~s}^{2} 2 \mathrm{p}^{2}{ }^{3} \mathrm{P}$} & $\overline{0}$ & 0 & 0 & 0 & & & \\
\hline & 1 & 29647 & 29658 & -11 & 29647 & 29658 & -11 \\
\hline & 2 & 55733 & 55730 & 3 & 55733 & 55730 & 3 \\
\hline $2 \mathrm{~s}^{2} 2 \mathrm{p}^{21} \mathrm{D}$ & 2 & 140771 & 140660 & 111 & & & \\
\hline $2 \mathrm{~s}^{2} 2 \mathrm{p}^{2}{ }^{1} \mathrm{~S}$ & 0 & 242313 & 242180 & 133 & & & \\
\hline $2 \mathrm{~s} 2 \mathrm{p}^{3}{ }^{5} \mathrm{~S}^{o}$ & 2 & 333487 & 333660 & -173 & & & \\
\hline \multirow[t]{3}{*}{$2 \mathrm{~s} 2 \mathrm{p}^{3}{ }^{3} \mathrm{D}^{o}$} & 2 & 578947 & 578890 & 57 & & & \\
\hline & 1 & 580190 & 580110 & 80 & 1243 & 1220 & 23 \\
\hline & 3 & 586836 & 586760 & 76 & 7889 & 7870 & 19 \\
\hline \multirow[t]{3}{*}{$2 \mathrm{~s} 2 \mathrm{p}^{3}{ }^{3} \mathrm{P}^{o}$} & 0 & 678667 & 678450 & 217 & & & \\
\hline & 1 & 681139 & 680910 & 229 & 2472 & 2460 & 12 \\
\hline & 2 & 686982 & 686780 & 202 & 8315 & 8330 & -15 \\
\hline $2 \mathrm{~s} 2 \mathrm{p}^{3}{ }^{3} \mathrm{~S}^{o}$ & 1 & 838569 & 838340 & 229 & & & \\
\hline $2 \mathrm{~s} 2 \mathrm{p}^{31} \mathrm{D}^{o}$ & 2 & 845454 & 845140 & 314 & & & \\
\hline $2 \mathrm{~s} 2 \mathrm{p}^{3}{ }^{1} \mathrm{P}^{o}$ & 1 & 943866 & 943500 & 366 & & & \\
\hline
\end{tabular}

involved. In the RCI calculations some QED effects, such as vacuum polarization and self-energy correction, can also be accounted for [29, 30]. Calculations can be done for individual levels, but also for subsets of a spectrum in the extended optimal level (EOL) scheme, where optimization is on a weighted sum of energies [31]. In the latter scheme a balanced description of all the states in an $L S$ term can be obtained in a single calculation.

\section{Computation of atomic properties}

Once the atomic state functions have been obtained, different properties like hyperfine structures, isotope shifts and oscillator strengths can be expressed in terms of reduced matrix elements of tensor operators of different rank

$$
\left\langle\Psi(\gamma J)\left\|\mathbf{T}^{(k)}\right\| \Psi\left(\gamma^{\prime} J^{\prime}\right)\right\rangle .
$$

After inserting the CSF expansions, the expression above reduces to a sum over matrix elements between CSFs. With the use of the Racah algebra techniques these matrix elements, in turn, can be obtained as a sum over radial integrals [27].
Table 4. Computed magnetic dipole hyperfine interaction constants in $\mathrm{MHz}$ for $2 \mathrm{~s}^{2} 2 \mathrm{p}^{2}{ }^{3} \mathrm{P}_{1,2}$ and $2 \mathrm{~s} 2 \mathrm{p}^{3}{ }^{3} \mathrm{P}_{1,2}^{o}$ of $\mathrm{F} \mathrm{IV}$, as functions of the increasing active sets, where $n=3$ denotes the orbital set with maximal principal quantum number $n=3$, etc. MR denotes calculations using the $n=8$ active set, but with an enlarged multireference set. The hyperfine constants are computed for ${ }^{19} \mathrm{~F}$ with $I=1 / 2, \mu=2.628868 \mathrm{~nm}$.

\begin{tabular}{lrllll}
\hline & \multicolumn{2}{c}{$2 \mathrm{~s}^{2} 2 \mathrm{p}^{2}{ }^{3} \mathrm{P}_{1,2}$} & & \multicolumn{2}{c}{$2 \mathrm{~s} 2 \mathrm{p}^{3}{ }^{3} \mathrm{P}_{1,2}^{o}$} \\
\cline { 2 - 3 } \cline { 5 - 6 } & \multicolumn{1}{l}{$A_{1}$} & $A_{2}$ & & & $A_{1}$ \\
\hline 3 & $-8.850+02$ & $2.796+03$ & & $2.234+04$ & $1.881+04$ \\
4 & $-2.813+02$ & $3.565+03$ & & $2.245+04$ & $1.875+04$ \\
5 & $3.952+01$ & $3.800+03$ & & $2.243+04$ & $1.875+04$ \\
6 & $-1.127+02$ & $3.674+03$ & & $2.238+04$ & $1.871+04$ \\
7 & $-6.741+01$ & $3.718+03$ & & $2.234+04$ & $1.867+04$ \\
8 & $-9.089+01$ & $3.702+03$ & & $2.235+04$ & $1.868+04$ \\
MR & $-7.992+01$ & $3.713+03$ & & $2.235+04$ & $1.867+04$ \\
\hline
\end{tabular}

\subsection{Isotope shift}

An isotope of an element is characterized by the mass $M$ and the mean-square nuclear radius $\left\langle r_{M}^{2}\right\rangle$. When effects of the finite nuclear mass and the extended nuclear charge distribution are 
Table 5. Hyperfine interaction constants $A_{J}$ and $B_{J}$ in MHz and Landé $g_{J}$-factors of N II, O III, F IV, Ne V and Ti XVII from multireference $\mathrm{RCI}$ calculations. The hyperfine constants are computed for: ${ }^{15} \mathrm{~N}$ with $I=1, \mu=0.40376100 \mathrm{~nm}, Q=0.02001 \mathrm{~b} ;{ }^{17} \mathrm{O}$ with $I=5 / 2$, $\mu=-1.89379 \mathrm{~nm}, Q=-0.02578 \mathrm{~b} ;{ }^{19} \mathrm{~F}$ with $I=1 / 2, \mu=2.628868 \mathrm{~nm} ;{ }^{21}$ Ne with $I=3 / 2, \mu=-0.661797 \mathrm{~nm}, Q=0.103 \mathrm{~b}$; and ${ }^{47}$ Ti with $I=5 / 2, \mu=-0.78848 \mathrm{~nm}, Q=0.290 \mathrm{~b}$.

\begin{tabular}{|c|c|c|c|c|c|c|c|}
\hline Level & $J$ & $A_{J}$ & $B_{J}$ & $g_{J}$ & $A_{J}$ & $B_{J}$ & $g_{J}$ \\
\hline & & & N II & & & O III & \\
\hline \multirow[t]{2}{*}{$2 \mathrm{~s}^{2} 2 \mathrm{p}^{2}{ }^{3} \mathrm{P}$} & 1 & $-1.229+00$ & $3.392+00$ & 1.50104 & $5.536+00$ & $-8.224+00$ & 1.50097 \\
\hline & 2 & $9.284+01$ & $-6.796+00$ & 1.50101 & $-3.227+02$ & $1.649+01$ & 1.50091 \\
\hline $2 \mathrm{~s}^{2} 2 \mathrm{p}^{21} \mathrm{D}$ & 2 & $1.443+02$ & $1.329+01$ & 0.99990 & $-5.080+02$ & $-3.220+01$ & 0.99985 \\
\hline $2 \mathrm{~s} 2 \mathrm{p}^{35} \mathrm{~S}^{o}$ & 2 & $4.539+02$ & $-6.579-05$ & 2.00215 & $-1.445+03$ & $1.924-04$ & 2.00206 \\
\hline \multirow[t]{3}{*}{$2 \mathrm{~s} 2 \mathrm{p}^{3}{ }^{3} \mathrm{D}^{o}$} & 3 & $4.288+02$ & $-7.700-01$ & 1.33397 & $-1.406+03$ & $1.093+00$ & 1.33389 \\
\hline & 2 & $2.394+02$ & $-2.809-01$ & 1.16695 & $-7.935+02$ & $8.806-02$ & .16690 \\
\hline & 1 & $-3.667+02$ & $-2.176-01$ & 0.49880 & $1.127+03$ & $1.541-01$ & 0.49879 \\
\hline \multirow[t]{2}{*}{$2 \mathrm{~s} 2 \mathrm{p}^{3}{ }^{3} \mathrm{P}^{o}$} & 2 & $5.222+02$ & $4.824-01$ & 1.50101 & $-1.698+03$ & $-4.481-01$ & 1.50092 \\
\hline & 1 & $6.128+02$ & $-3.496-01$ & 1.50099 & $-2.015+03$ & $6.904-01$ & 1.50087 \\
\hline $2 \mathrm{~s} 2 \mathrm{p}^{31} \mathrm{D}^{o}$ & 2 & $1.379+02$ & $-1.258-01$ & 0.99989 & $-4.975+02$ & $4.537-01$ & 0.99983 \\
\hline $2 \mathrm{~s} 2 \mathrm{p}^{3}{ }^{3} \mathrm{~S}^{o}$ & 1 & - & - & - & $1.741+03$ & $3.024-04$ & 2.00192 \\
\hline \multirow[t]{2}{*}{$2 \mathrm{~s} 2 \mathrm{p}^{3}{ }^{1} \mathrm{P}^{o}$} & 1 & - & - & - & $-5.102+02$ & $-6.827-01$ & 0.99998 \\
\hline & & & F IV & & & $\mathrm{Ne} \mathrm{V}$ & \\
\hline \multirow[t]{2}{*}{$2 \mathrm{~s}^{2} 2 \mathrm{p}^{23} \mathrm{P}$} & 1 & $-7.996+01$ & & 1.50089 & $1.187+01$ & $8.519+01$ & 1.50080 \\
\hline & 2 & $3.713+03$ & & 1.50076 & $-4.800+02$ & $-1.709+02$ & 1.50055 \\
\hline $2 \mathrm{~s}^{2} 2 \mathrm{p}^{21} \mathrm{D}$ & 2 & $5.885+03$ & & 0.99982 & $-7.615+02$ & $3.360+02$ & 0.99983 \\
\hline $2 \mathrm{~s} 2 \mathrm{p}^{35} \mathrm{~S}^{o}$ & 2 & $1.575+04$ & & 2.00195 & $-1.959+03$ & $-4.281-03$ & 2.00182 \\
\hline \multirow[t]{3}{*}{$2 \mathrm{~s} 2 \mathrm{p}^{3}{ }^{3} \mathrm{D}^{o}$} & 3 & $1.561+04$ & & 1.33380 & $-1.965+03$ & $-5.287+00$ & 1.33370 \\
\hline & 2 & $8.871+03$ & & 1.16686 & $-1.122+03$ & $9.321+00$ & 1.16685 \\
\hline & 1 & $-1.196+04$ & & 0.49881 & $1.455+03$ & $4.125+00$ & 0.49890 \\
\hline \multirow[t]{2}{*}{$2 \mathrm{~s} 2 \mathrm{p}^{3}{ }^{3} \mathrm{P}^{o}$} & 2 & $1.867+04$ & & 1.50078 & $-2.332+03$ & $-6.820+00$ & 1.50059 \\
\hline & 1 & $2.235+04$ & & 1.50071 & $-2.808+03$ & $-8.641+00$ & 1.50047 \\
\hline $2 \mathrm{~s} 2 \mathrm{p}^{3}{ }^{1} \mathrm{D}^{o}$ & 2 & $5.808+03$ & & 0.99976 & $-7.561+02$ & $-7.099+00$ & 0.99969 \\
\hline $2 \mathrm{~s} 2 \mathrm{p}^{3}{ }^{3} \mathrm{~S}^{o}$ & 1 & $-1.874+04$ & & 2.00164 & $2.300+03$ & $-1.057-02$ & 2.00119 \\
\hline \multirow[t]{2}{*}{$2 \mathrm{~s} 2 \mathrm{p}^{3}{ }^{1} \mathrm{P}^{o}$} & 1 & $5.857+03$ & & 1.00007 & $-7.567+02$ & $6.092+00$ & 1.00029 \\
\hline & & & Ti XVII & & & & \\
\hline \multirow{2}{*}{$2 \mathrm{~s}^{2} 2 \mathrm{p}^{2}{ }^{3} \mathrm{P}$} & 1 & $3.422+02$ & $4.271+03$ & 1.49 & & & \\
\hline & 2 & $-7.593+03$ & $-6.135+03$ & 1.447 & & & \\
\hline $2 s^{2} 2 p^{2}{ }^{1} D$ & 2 & $-8.075+03$ & $1.458+04$ & 1.04823 & & & \\
\hline $2 \mathrm{~s} 2 \mathrm{p}^{3}{ }^{5} \mathrm{~S}^{o}$ & 2 & $-2.190+04$ & $2.631+01$ & 1.99313 & & & \\
\hline \multirow[t]{3}{*}{$2 \mathrm{~s} 2 \mathrm{p}^{3}{ }^{3} \mathrm{D}^{o}$} & 3 & $-2.272+04$ & $-2.958+01$ & 1.33122 & & & \\
\hline & 2 & $-1.493+04$ & $8.403+03$ & 1.19492 & & & \\
\hline & 1 & $1.351+04$ & $4.592+03$ & 0.55917 & & & \\
\hline \multirow[t]{2}{*}{$2 \mathrm{~s} 2 \mathrm{p}^{3}{ }^{3} \mathrm{P}^{o}$} & 2 & $-2.320+04$ & $-8.321+03$ & 1.46142 & & & \\
\hline & 1 & $-2.992+04$ & $-4.644+03$ & 1.44806 & & & \\
\hline $2 \mathrm{~s} 2 \mathrm{p}^{3}{ }^{1} \mathrm{D}^{o}$ & 2 & $-1.077+04$ & $-3.029+02$ & 1.00997 & & & \\
\hline $2 \mathrm{~s} 2 \mathrm{p}^{3}{ }^{3} \mathrm{~S}^{o}$ & 1 & $1.788+04$ & $2.995+01$ & 1.90611 & & & \\
\hline $2 \mathrm{~s} 2 \mathrm{p}^{3}{ }^{1} \mathrm{P}^{o}$ & 1 & $-4.729+03$ & $1.389+02$ & 1.07843 & & & \\
\hline
\end{tabular}

Table 6. Computed specific mass shift parameters $S_{\text {sms }}$ in au and electron densities $\rho(0)$ at the origin for $2 \mathrm{~s}^{2} 2 \mathrm{p}^{2}{ }^{3} \mathrm{P}_{0}$ and $2 \mathrm{~s} 2 \mathrm{p}^{3}{ }^{3} \mathrm{P}_{0}^{o}$ in F IV as functions of the increasing active sets where $n=3$ denotes the set $3 \mathrm{~s} 2 \mathrm{p} 1 \mathrm{~d}$ etc. MR denotes calculations using the $n=8$ active set, but with an enlarged multireference set.

\begin{tabular}{|c|c|c|c|c|c|c|}
\hline \multirow[b]{2}{*}{$n$} & \multicolumn{2}{|c|}{$2 \mathrm{~s}^{2} 2 \mathrm{p}^{2}{ }^{3} \mathrm{P}_{0}$} & \multicolumn{2}{|c|}{$2 \mathrm{~s} 2 \mathrm{p}^{3}{ }^{3} \mathrm{P}_{0}^{o}$} & \multicolumn{2}{|c|}{$2 \mathrm{~s} 2 \mathrm{p}^{3}{ }^{3} \mathrm{P}_{0}^{o}-2 \mathrm{~s}^{2} 2 \mathrm{p}^{2}{ }^{3} \mathrm{P}_{0}$} \\
\hline & $S_{\mathrm{sms}}$ & $\rho(0)$ & $\overline{S_{\mathrm{sms}}}$ & $\rho(0)$ & $\overline{\Delta S_{\mathrm{sms}}}$ & $\Delta \rho(0)$ \\
\hline 3 & -3.938 & 470.635 & -6.233 & 455.803 & -2.295 & -14.832 \\
\hline 4 & -4.012 & 470.619 & -6.302 & 455.767 & -2.290 & -14.852 \\
\hline 5 & -4.006 & 470.684 & -6.308 & 455.795 & -2.302 & -14.889 \\
\hline 6 & -4.004 & 470.709 & -6.304 & 455.849 & -2.300 & -14.860 \\
\hline 7 & -4.009 & 470.722 & -6.306 & 455.869 & -2.297 & -14.853 \\
\hline 8 & -4.010 & 470.734 & -6.308 & 455.891 & -2.298 & -14.843 \\
\hline MR & -3.984 & 470.733 & -6.277 & 455.913 & -2.293 & -14.820 \\
\hline
\end{tabular}


Table 7. Specific mass shift parameters $S_{\mathrm{sms}}$ in au and electron densities $\rho(0)$ at the origin for N II, O III, F IV, Ne V and Ti XVII from multireference RCI calculations.

\begin{tabular}{|c|c|c|c|c|c|c|c|}
\hline Level & $J$ & $S_{\mathrm{sms}}$ & $\rho(0)$ & $S_{\text {sms }}$ & $\rho(0)$ & $S_{\mathrm{sms}}$ & $\rho(0)$ \\
\hline & & \multicolumn{2}{|c|}{ N II } & \multicolumn{2}{|c|}{ O III } & \multicolumn{2}{|c|}{ F IV } \\
\hline \multirow{3}{*}{$2 \mathrm{~s}^{2} 2 \mathrm{p}^{23} \mathrm{P}$} & 0 & -1.280 & 211.42 & -2.473 & 323.50 & -3.984 & 470.73 \\
\hline & 1 & -1.279 & 211.42 & -2.471 & 323.50 & -3.981 & 470.73 \\
\hline & 2 & -1.278 & 211.42 & -2.468 & 323.50 & -3.977 & 470.73 \\
\hline $2 \mathrm{~s}^{2} 2 \mathrm{p}^{21} \mathrm{D}$ & 2 & -1.236 & 211.52 & -2.422 & 323.61 & -3.926 & 470.85 \\
\hline $2 \mathrm{~s}^{2} 2 \mathrm{p}^{2}{ }^{1} \mathrm{~S}$ & 0 & -1.230 & 211.31 & -2.415 & 323.29 & -3.924 & 470.31 \\
\hline $2 \mathrm{~s} 2 \mathrm{p}^{3}{ }^{5} \mathrm{~S}^{o}$ & 2 & -2.487 & 206.29 & -4.323 & 314.49 & -6.631 & 456.27 \\
\hline \multirow[t]{3}{*}{$2 \mathrm{~s} 2 \mathrm{p}^{3}{ }^{3} \mathrm{D}^{o}$} & 3 & -2.387 & 206.25 & -4.208 & 314.29 & -6.506 & 455.88 \\
\hline & 2 & -2.387 & 206.25 & -4.208 & 314.29 & -6.505 & 455.88 \\
\hline & 1 & -2.387 & 206.25 & -4.208 & 314.30 & -6.504 & 455.88 \\
\hline \multirow[t]{3}{*}{$2 \mathrm{~s} 2 \mathrm{p}^{3}{ }^{3} \mathrm{P}^{o}$} & 2 & -2.250 & 206.31 & -4.025 & 314.33 & -6.277 & 455.91 \\
\hline & 1 & -2.250 & 206.31 & -4.025 & 314.33 & -6.277 & 455.91 \\
\hline & 0 & -2.250 & 206.31 & -4.026 & 314.33 & -6.277 & 455.91 \\
\hline $2 \mathrm{~s} 2 \mathrm{p}^{31} \mathrm{D}^{o}$ & 2 & -2.127 & 206.00 & -3.918 & 313.50 & -6.199 & 454.57 \\
\hline $2 \mathrm{~s} 2 \mathrm{p}^{3}{ }^{3} \mathrm{~S}^{o}$ & 1 & - & - & -3.592 & 312.64 & -5.895 & 453.47 \\
\hline \multirow[t]{2}{*}{$2 \mathrm{~s} 2 \mathrm{p}^{3}{ }^{1} \mathrm{P}^{o}$} & 1 & - & - & -3.569 & 313.50 & -5.830 & 454.53 \\
\hline & & \multicolumn{2}{|c|}{$\mathrm{NeV}$} & \multicolumn{2}{|c|}{ Ti XVII } & & \\
\hline \multirow[t]{3}{*}{$2 \mathrm{~s}^{2} 2 \mathrm{p}^{23} \mathrm{P}$} & 0 & -5.816 & 658.51 & -53.279 & 8536.04 & & \\
\hline & 1 & -5.810 & 658.51 & -52.831 & 8535.95 & & \\
\hline & 2 & -5.802 & 658.51 & -52.647 & 8535.23 & & \\
\hline $2 \mathrm{~s}^{2} 2 \mathrm{p}^{21} \mathrm{D}$ & 2 & -5.748 & 658.64 & -52.393 & 8534.76 & & \\
\hline $2 s^{2} 2 p^{2}{ }^{1} S$ & 0 & -5.758 & 657.63 & -52.897 & 8516.67 & & \\
\hline $2 \mathrm{~s} 2 \mathrm{p}^{3}{ }^{5} \mathrm{~S}^{o}$ & 2 & -9.411 & 636.81 & -80.082 & 8146.94 & & \\
\hline \multirow[t]{3}{*}{$2 \mathrm{~s} 2 \mathrm{p}^{3}{ }^{3} \mathrm{D}^{o}$} & 3 & -9.277 & 636.18 & -79.832 & 8139.90 & & \\
\hline & 2 & -9.276 & 636.18 & -79.937 & 8140.17 & & \\
\hline & 1 & -9.276 & 636.18 & -79.936 & 8140.05 & & \\
\hline \multirow[t]{3}{*}{$2 \mathrm{~s} 2 \mathrm{p}^{3}{ }^{3} \mathrm{P}^{o}$} & 2 & -9.002 & 636.21 & -78.863 & 8139.37 & & \\
\hline & 1 & -9.001 & 636.21 & -78.937 & 8139.53 & & \\
\hline & 0 & -9.001 & 636.21 & -78.946 & 8139.95 & & \\
\hline $2 \mathrm{~s} 2 \mathrm{p}^{31} \mathrm{D}^{o}$ & 2 & -8.961 & 634.31 & -79.407 & 8124.45 & & \\
\hline $2 \mathrm{~s} 2 \mathrm{p}^{3}{ }^{3} \mathrm{~S}^{o}$ & 1 & -8.674 & 632.92 & -79.405 & 8116.68 & & \\
\hline $2 \mathrm{~s} 2 \mathrm{p}^{3}{ }^{1} \mathrm{P}^{o}$ & 1 & -8.556 & 634.26 & -78.355 & 8123.24 & & \\
\hline
\end{tabular}

taken into account, the energy of a level $\gamma J$ can be written approximately as

$E_{M}=E_{0}-E_{0} \frac{m}{M+m}+S_{\mathrm{sms}} \frac{M}{(M+m)^{2}}+\frac{2}{3} \pi Z \rho(0)\left\langle r_{M}^{2}\right\rangle$

where $E_{0}$ is the calculated energy for infinite mass, $S_{\mathrm{sms}}$ is the specific mass shift parameter

$$
S_{\mathrm{sms}}=\left\langle\Psi\left(\gamma J M_{J}\right)\left|\sum_{i<j}^{N} \mathbf{p}_{i} \cdot \mathbf{p}_{j}\right| \Psi\left(\gamma J M_{J}\right)\right\rangle,
$$

and $\rho(0)$ the electron density at the site of the nucleus

$$
\rho(0)=\left\langle\Psi\left(\gamma J M_{J}\right)\left|\sum_{i=1}^{N} \delta\left(\mathbf{r}_{i}\right)\right| \Psi\left(\gamma J M_{J}\right)\right\rangle .
$$

The isotopic shift of an individual level is not a directly measurable quantity. What can be measured is the difference in transition energy between an upper and a lower level for different isotopes. Let $\Delta E_{0}$ be the transition energy between an upper and a lower level for infinite mass. The difference in transition energy between two isotopes with masses $M^{\prime}$ and $M$ is then given by

$$
\begin{gathered}
\Delta E_{M^{\prime}, M}=\Delta E_{0}\left[\frac{m}{M+m}-\frac{m}{M^{\prime}+m}\right] \\
+\Delta S_{\text {sms }}\left[\frac{M^{\prime}}{\left(M^{\prime}+m\right)^{2}}-\frac{M}{(M+m)^{2}}\right] \\
+\frac{2}{3} \pi Z \Delta \rho(0)\left[\left\langle r_{M^{\prime}}^{2}\right\rangle-\left\langle r_{M}^{2}\right\rangle\right],
\end{gathered}
$$

where the differences in the electronic parameters are between the values of the upper and lower levels in the transition. For light elements the transition isotope shift is dominated by the mass shift [32]. In practical calculations $\Delta E_{0}$ is often replaced by the experimental transition energy (rather than the calculated transition energy for the infinite mass). All quantities in the above formulas are expressed in atomic units (au). The nuclear masses $M$ and $M^{\prime}$ are usually given in units of the unified atomic mass (u) and must be converted to atomic units. We have used $1 \mathrm{u}=1822.88851 \mathrm{au}$.

\subsection{Hyperfine structure and Landé factors}

The fine-structure levels are split into closely spaced hyperfine levels in atoms with non-zero nuclear spin. The hyperfine 
Table 8. Transition isotope shifts in $\mathrm{cm}^{-1}$ in N II, O III and Ne V. A positive sign of the transition isotope shift corresponds to a larger energy transition (i.e. a blue shifted line) for the heavier isotope.

\begin{tabular}{|c|c|c|c|c|c|c|}
\hline \multicolumn{2}{|c|}{ Levels } & \multicolumn{5}{|c|}{ Isotopic pair } \\
\hline Upper & Lower & $\overline{15,14} \mathrm{~N}$ & ${ }^{18,16} \mathrm{O}$ & ${ }^{17,16} \mathrm{O}$ & ${ }^{22,20} \mathrm{Ne}$ & ${ }^{21,20} \mathrm{Ne}$ \\
\hline $2 \mathrm{~s} 2 \mathrm{p}^{3}{ }^{3} \mathrm{D}_{1}^{o}$ & $2 \mathrm{~s}^{2} 2 \mathrm{p}^{2}{ }^{3} \mathrm{P}_{0}$ & 0.873 & 1.914 & 1.015 & 2.334 & 1.225 \\
\hline $2 \mathrm{~s} 2 \mathrm{p}^{3}{ }^{3} \mathrm{P}_{1}^{o}$ & $2 \mathrm{~s}^{2} 2 \mathrm{p}^{2}{ }^{3} \mathrm{P}_{0}$ & 0.839 & 1.846 & 0.979 & 2.264 & 1.188 \\
\hline $2 \mathrm{~s} 2 \mathrm{p}^{33} \mathrm{~S}_{1}^{o}$ & $2 \mathrm{~s}^{2} 2 \mathrm{p}^{2}{ }^{3} \mathrm{P}_{0}$ & - & 1.696 & 0.890 & 2.263 & 1.187 \\
\hline $2 \mathrm{~s} 2 \mathrm{p}^{3}{ }^{1} \mathrm{P}_{1}^{o}$ & $2 \mathrm{~s}^{2} 2 \mathrm{p}^{2}{ }^{3} \mathrm{P}_{0}$ & - & 1.724 & 0.915 & 2.259 & 1.186 \\
\hline $2 \mathrm{~s} 2 \mathrm{p}^{35} \mathrm{~S}_{2}^{o}$ & $2 \mathrm{~s}^{2} 2 \mathrm{p}^{2}{ }^{3} \mathrm{P}_{1}$ & 0.812 & 1.783 & 0.946 & 2.192 & 1.150 \\
\hline $2 \mathrm{~s} 2 \mathrm{p}^{3}{ }^{3} \mathrm{D}_{2}^{o}$ & $2 \mathrm{~s}^{2} 2 \mathrm{p}^{2}{ }^{3} \mathrm{P}_{1}$ & 0.874 & 1.915 & 1.016 & 2.336 & 1.226 \\
\hline $2 \mathrm{~s} 2 \mathrm{p}^{3}{ }^{3} \mathrm{D}_{1}^{o}$ & $2 \mathrm{~s}^{2} 2 \mathrm{p}^{2}{ }^{3} \mathrm{P}_{1}$ & 0.874 & 1.915 & 1.016 & 2.336 & 1.226 \\
\hline $2 \mathrm{~s} 2 \mathrm{p}^{3}{ }^{3} \mathrm{P}_{2}^{o}$ & $2 \mathrm{~s}^{2} 2 \mathrm{p}^{2}{ }^{3} \mathrm{P}_{1}$ & 0.839 & 1.847 & 0.980 & 2.266 & 1.189 \\
\hline $2 \mathrm{~s} 2 \mathrm{p}^{3}{ }^{3} \mathrm{P}_{1}^{o}$ & $2 \mathrm{~s}^{2} 2 \mathrm{p}^{2}{ }^{3} \mathrm{P}_{1}$ & 0.839 & 1.847 & 0.980 & 2.266 & 1.189 \\
\hline $2 \mathrm{~s} 2 \mathrm{p}^{3}{ }^{3} \mathrm{P}_{0}^{o}$ & $2 \mathrm{~s}^{2} 2 \mathrm{p}^{2}{ }^{3} \mathrm{P}_{1}$ & 0.840 & 1.847 & 0.980 & 2.266 & 1.189 \\
\hline $2 \mathrm{~s} 2 \mathrm{p}^{31}{ }^{1} \mathrm{D}_{2}^{o}$ & $2 \mathrm{~s}^{2} 2 \mathrm{p}^{2}{ }^{3} \mathrm{P}_{1}$ & 0.861 & 1.928 & 1.023 & 2.400 & 1.259 \\
\hline $2 \mathrm{~s} 2 \mathrm{p}^{3}{ }^{3} \mathrm{~S}_{1}^{o}$ & $2 \mathrm{~s}^{2} 2 \mathrm{p}^{2}{ }^{3} \mathrm{P}_{1}$ & - & 1.693 & 0.898 & 2.265 & 1.188 \\
\hline $2 \mathrm{~s} 2 \mathrm{p}^{3}{ }^{1} \mathrm{P}_{1}^{o}$ & $2 \mathrm{~s}^{2} 2 \mathrm{p}^{2}{ }^{3} \mathrm{P}_{1}$ & - & 1.725 & 0.915 & 2.261 & 1.187 \\
\hline $2 \mathrm{~s} 2 \mathrm{p}^{35} \mathrm{~S}_{2}^{o}$ & $2 \mathrm{~s}^{2} 2 \mathrm{p}^{2}{ }^{3} \mathrm{P}_{2}$ & 0.813 & 1.784 & 0.947 & 2.194 & 1.151 \\
\hline $2 \mathrm{~s} 2 \mathrm{p}^{3}{ }^{3} \mathrm{D}_{3}^{o}$ & $2 \mathrm{~s}^{2} 2 \mathrm{p}^{2}{ }^{3} \mathrm{P}_{2}$ & 0.874 & 1.916 & 1.017 & 2.339 & 1.228 \\
\hline $2 \mathrm{~s} 2 \mathrm{p}^{3}{ }^{3} \mathrm{D}_{2}^{o}$ & $2 \mathrm{~s}^{2} 2 \mathrm{p}^{2}{ }^{3} \mathrm{P}_{2}$ & 0.874 & 1.916 & 1.017 & 2.339 & 1.227 \\
\hline $2 \mathrm{~s} 2 \mathrm{p}^{3}{ }^{3} \mathrm{D}_{1}^{o}$ & $2 \mathrm{~s}^{2} 2 \mathrm{p}^{2}{ }^{3} \mathrm{P}_{2}$ & 0.874 & 1.916 & 1.017 & 2.339 & 1.227 \\
\hline $2 \mathrm{~s} 2 \mathrm{p}^{3}{ }^{3} \mathrm{P}_{2}^{o}$ & $2 \mathrm{~s}^{2} 2 \mathrm{p}^{2}{ }^{3} \mathrm{P}_{2}$ & 0.840 & 1.848 & 0.981 & 2.269 & 1.191 \\
\hline $2 \mathrm{~s} 2 \mathrm{p}^{3}{ }^{3} \mathrm{P}_{1}^{o}$ & $2 \mathrm{~s}^{2} 2 \mathrm{p}^{2}{ }^{3} \mathrm{P}_{2}$ & 0.840 & 1.848 & 0.980 & 2.269 & 1.191 \\
\hline $2 \mathrm{~s} 2 \mathrm{p}^{3}{ }^{1} \mathrm{D}_{2}^{o}$ & $2 \mathrm{~s}^{2} 2 \mathrm{p}^{2}{ }^{3} \mathrm{P}_{2}$ & 0.861 & 1.929 & 1.023 & 2.403 & 1.261 \\
\hline $2 \mathrm{~s} 2 \mathrm{p}^{3}{ }^{3} \mathrm{~S}_{1}^{o}$ & $2 \mathrm{~s}^{2} 2 \mathrm{p}^{2}{ }^{3} \mathrm{P}_{2}$ & - & 1.694 & 0.899 & 2.267 & 1.190 \\
\hline $2 \mathrm{~s} 2 \mathrm{p}^{3}{ }^{1} \mathrm{P}_{1}^{o}$ & $2 \mathrm{~s}^{2} 2 \mathrm{p}^{2}{ }^{3} \mathrm{P}_{2}$ & - & 1.726 & 0.916 & 2.264 & 1.188 \\
\hline $2 \mathrm{~s} 2 \mathrm{p}^{35} \mathrm{~S}_{2}^{o}$ & $2 \mathrm{~s}^{2} 2 \mathrm{p}^{2}{ }^{1} \mathrm{D}_{2}$ & 0.797 & 1.747 & 0.927 & 2.151 & 1.129 \\
\hline $2 \mathrm{~s} 2 \mathrm{p}^{3}{ }^{3} \mathrm{D}_{3}^{o}$ & $2 \mathrm{~s}^{2} 2 \mathrm{p}^{2}{ }^{1} \mathrm{D}_{2}$ & 0.858 & 1.879 & 0.997 & 2.296 & 1.205 \\
\hline $2 \mathrm{~s} 2 \mathrm{p}^{3}{ }^{3} \mathrm{D}_{2}^{o}$ & $2 \mathrm{~s}^{2} 2 \mathrm{p}^{2}{ }^{1} \mathrm{D}_{2}$ & 0.858 & 1.879 & 0.997 & 2.296 & 1.205 \\
\hline $2 \mathrm{~s} 2 \mathrm{p}^{3}{ }^{3} \mathrm{D}_{1}^{o}$ & $2 \mathrm{~s}^{2} 2 \mathrm{p}^{21} \mathrm{D}_{2}$ & 0.858 & 1.879 & 0.997 & 2.295 & 1.205 \\
\hline $2 \mathrm{~s} 2 \mathrm{p}^{3}{ }^{3} \mathrm{P}_{2}^{o}$ & $2 \mathrm{~s}^{2} 2 \mathrm{p}^{2}{ }^{1} \mathrm{D}_{2}$ & 0.824 & 1.811 & 0.961 & 2.226 & 1.168 \\
\hline $2 \mathrm{~s} 2 \mathrm{p}^{3}{ }^{3} \mathrm{P}_{1}^{o}$ & $2 \mathrm{~s}^{2} 2 \mathrm{p}^{2}{ }^{1} \mathrm{D}_{2}$ & 0.824 & 1.811 & 0.961 & 2.226 & 1.168 \\
\hline $2 \mathrm{~s} 2 \mathrm{p}^{3}{ }^{1} \mathrm{D}_{2}^{o}$ & $2 \mathrm{~s}^{2} 2 \mathrm{p}^{2}{ }^{1} \mathrm{D}_{2}$ & 0.845 & 1.892 & 1.004 & 2.359 & 1.238 \\
\hline $2 \mathrm{~s} 2 \mathrm{p}^{3}{ }^{3} \mathrm{~S}_{1}^{o}$ & $2 \mathrm{~s}^{2} 2 \mathrm{p}^{2}{ }^{1} \mathrm{D}_{2}$ & - & 1.657 & 0.879 & 2.224 & 1.167 \\
\hline $2 \mathrm{~s} 2 \mathrm{p}^{3}{ }^{1} \mathrm{P}_{1}^{o}$ & $2 \mathrm{~s}^{2} 2 \mathrm{p}^{2}{ }^{1} \mathrm{D}_{2}$ & - & 1.689 & 0.896 & 2.220 & 1.165 \\
\hline $2 \mathrm{~s} 2 \mathrm{p}^{3}{ }^{3} \mathrm{D}_{1}^{o}$ & $2 \mathrm{~s}^{2} 2 \mathrm{p}^{2}{ }^{1} \mathrm{~S}_{0}$ & 0.816 & 1.797 & 0.953 & 2.206 & 1.158 \\
\hline $2 \mathrm{~s} 2 \mathrm{p}^{3}{ }^{3} \mathrm{P}_{1}^{o}$ & $2 \mathrm{~s}^{2} 2 \mathrm{p}^{2}{ }^{1} \mathrm{~S}_{0}$ & 0.782 & 1.729 & 0.917 & 2.136 & 1.121 \\
\hline $2 \mathrm{~s} 2 \mathrm{p}^{3}{ }^{3} \mathrm{~S}_{1}^{o}$ & $2 \mathrm{~s}^{2} 2 \mathrm{p}^{2}{ }^{1} \mathrm{~S}_{0}$ & - & 1.575 & 0.836 & 2.135 & 1.120 \\
\hline $2 \mathrm{~s} 2 \mathrm{p}^{3}{ }^{1} \mathrm{P}_{1}^{o}$ & $2 \mathrm{~s}^{2} 2 \mathrm{p}^{2}{ }^{1} \mathrm{~S}_{0}$ & - & 1.607 & 0.852 & 2.131 & 1.118 \\
\hline
\end{tabular}

shifts of the fine-structure levels may be to first order expressed in terms of magnetic dipole $A_{J}$ and electric quadrupole $B_{J}$ hyperfine interaction constants

$$
\begin{aligned}
A_{J} & =\frac{\mu_{I}}{I} \frac{1}{\sqrt{J(J+1)(2 J+1)}} \\
& \times\left\langle\Psi(\gamma J)\left\|-\sum_{j=1}^{N} \mathrm{i} \sqrt{2} \alpha r_{j}^{-2}\left[\boldsymbol{\alpha}_{j} \mathbf{C}_{j}^{(1)}\right]^{(1)}\right\| \Psi(\gamma J)\right\rangle \\
B_{J} & =2 Q \sqrt{\frac{J(2 J-1)}{(J+1)(2 J+1)(2 J+3)}} \\
& \times\left\langle\Psi(\gamma J)\left\|-\sum_{j=1}^{N} r_{j}^{-3} \mathbf{C}_{j}^{(2)}\right\| \Psi(\gamma J)\right\rangle
\end{aligned}
$$

The hyperfine shifts of fine-structure levels are affected also by the off-diagonal hyperfine interaction [33]. However, this effect is small and has been neglected in the present study. The nuclear magnetic dipole moments $\mu_{I}$ and the nuclear quadrupole moments $Q$ for the different isotopes were taken from a recent compilation by Stone [34].

The Landé $g_{J}$-factor determines the splitting of magnetic sub-levels in external magnetic fields and gives information about the coupling conditions [35]. The Landé $g_{J}$-factor has a tensorial form similar to the one for magnetic hyperfine interaction and it was calculated using a modified version of the hyperfine structure program [15].

\subsection{Transition parameters}

The transition parameters, such as rates of spontaneous decay, for electric dipole transitions between two atomic states $\gamma J M_{J}$ and $\gamma^{\prime} J^{\prime} M_{J}^{\prime}$ can be expressed in terms of the reduced transition matrix element

$$
\left\langle\Psi(\gamma J)\left\|\mathbf{D}^{(1)}\right\| \Psi\left(\gamma^{\prime} J^{\prime}\right)\right\rangle,
$$

where $\mathrm{D}_{q}^{(1)}$ is the electromagnetic dipole operator in the Coulomb or Babushkin gauge [36]. Standard Racah algebra 
Table 9. Transition rates (Babushkin and Coulomb gauge) in $\mathrm{s}^{-1}$ for $2 \mathrm{~s}^{2} 2 \mathrm{p}^{2}{ }^{3} \mathrm{P}_{0}-2 \mathrm{~s} 2 \mathrm{p}^{3}{ }^{3} \mathrm{P}_{1}^{o}$ and $2 \mathrm{~s}^{2} 2 \mathrm{p}^{2}{ }^{1} \mathrm{D}_{2}-2 \mathrm{~s} 2 \mathrm{p}^{3}{ }^{1} \mathrm{P}_{1}^{o}$ in F IV as functions of the active sets, where $n=3$ denotes the orbital set with maximal principal quantum number $n=3$, etc. MR denotes calculations using the $n=8$ active set, but with an enlarged multireference set. Transition energies $\Delta E$ are given in units of $\mathrm{cm}^{-1}$. The experimental energies are those of [40].

\begin{tabular}{llllllll}
\hline & \multicolumn{3}{c}{$2 \mathrm{~s}^{2} 2 \mathrm{p}^{2}{ }^{3} \mathrm{P}_{0}-2 \mathrm{~s} 2 \mathrm{p}^{33} \mathrm{P}_{1}^{o}$} & & \multicolumn{3}{c}{$2 \mathrm{~s}^{2} 2 \mathrm{p}^{2}{ }^{1} \mathrm{D}_{2}-2 \mathrm{~s} 2 \mathrm{p}^{3}{ }^{1} \mathrm{P}_{1}^{o}$} \\
\cline { 2 - 4 } \cline { 6 - 8 }$n$ & $A_{B}$ & $A_{C}$ & $\Delta E$ & & $A_{B}$ & $A_{C}$ & $\Delta E$ \\
\hline 3 & $8.10+08$ & $7.82+08$ & 179447 & & $1.11+10$ & $9.50+09$ & 238047 \\
4 & $8.54+08$ & $8.51+08$ & 178036 & & $1.11+10$ & $1.04+10$ & 234333 \\
5 & $8.32+08$ & $8.30+08$ & 176006 & & $1.12+10$ & $1.06+10$ & 233489 \\
6 & $8.29+08$ & $8.22+08$ & 175712 & & $1.12+10$ & $1.06+10$ & 233139 \\
7 & $8.33+08$ & $8.26+08$ & 175592 & & $1.12+10$ & $1.07+10$ & 232996 \\
8 & $8.27+08$ & $8.15+08$ & 175562 & & $1.12+10$ & $1.07+10$ & 232923 \\
MR & $8.18+08$ & $8.15+08$ & 175513 & & $1.12+10$ & $1.11+10$ & 232562 \\
Exp & & & 175237 & & & & 232148 \\
\hline
\end{tabular}

Table 10. Transition rates (Babushkin and Coulomb gauge) in $\mathrm{s}^{-1}$ for N II from multireference RCI calculations. $A_{\mathrm{MCHF}}$ transition rates from [21]. Transition energies $\Delta E_{\exp }$ are given in units of $\mathrm{cm}^{-1}$ and are those of [40].

\begin{tabular}{|c|c|c|c|c|c|}
\hline pper & Lower & $E_{\text {exp }}$ & $A_{B}$ & $A_{C}$ & $\mathrm{IF}$ \\
\hline & & 51.80 & & 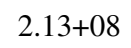 & $2.095+08$ \\
\hline & & & & & $4.226+08$ \\
\hline $28 n^{3}$ & $29 n^{2} 3 D$ & & 17 & 16.0 & $5.155+01$ \\
\hline & & & & & $.823+08$ \\
\hline & & 922 & $6+08$ & +08 & $1.545+08$ \\
\hline & & 109 & $6+08$ & $01+08$ & $3.139+08$ \\
\hline $\mathrm{P}_{1}^{o}$ & & 109 & $3+08$ & 18 & $3.214+08$ \\
\hline & & 09 & 9 & -09 & $1.273+09$ \\
\hline $2 \mathrm{~s} 2$ & & 44 & & & $5.657+03$ \\
\hline & & & & & \\
\hline & & & & & +08 \\
\hline $3^{3} \mathrm{D}_{2}^{o}$ & $2 \mathrm{~s}^{2} 2 \mathrm{p}^{2}{ }^{3} \mathrm{P}_{2}$ & 0 & & & $9.097+07$ \\
\hline $2 \mathrm{~s} 2 \mathrm{p}^{3}{ }^{3} \mathrm{D}_{1}^{o}$ & $2 \mathrm{~s}^{2} 2 \mathrm{p}^{2}{ }^{3} \mathrm{P}_{2}$ & 92 & & & $9.963+06$ \\
\hline $2 \mathrm{~s} 2 \mathrm{p}^{3}{ }^{3} \mathrm{P}_{2}^{o}$ & $2 \mathrm{~s}^{2} 2 \mathrm{p}^{2}{ }^{3} \mathrm{P}_{2}$ & 109 & & & $9.548+08$ \\
\hline $2 \mathrm{~s} 2 \mathrm{p}^{3}{ }^{3} \mathrm{P}_{1}^{o}$ & $2 \mathrm{~s}^{2} 2 \mathrm{p}^{2}{ }^{3} \mathrm{P}_{2}$ & 10 & & & $5.272+08$ \\
\hline $2 \mathrm{~s} 2 \mathrm{p}^{3}{ }^{1} \mathrm{D}_{2}^{o}$ & $2 \mathrm{~s}^{2} 2 \mathrm{p}^{2}{ }^{3} \mathrm{P}_{2}$ & & & & $4.141+04$ \\
\hline $\mathrm{s} 2 \mathrm{p}^{35} \mathrm{~S}_{2}^{o}$ & 20 & & & & -04 \\
\hline $2 \mathrm{~s} 2 \mathrm{p}^{33} \mathrm{D}_{3}^{o}$ & $2 \mathrm{~s}^{2} 2 \mathrm{p}^{2}{ }^{1} \mathrm{D}_{2}$ & & & & +03 \\
\hline $2 \mathrm{~s} 2 \mathrm{p}^{3}{ }^{3} \mathrm{D}_{2}^{o}$ & $2 \mathrm{~s}^{2} 2 \mathrm{p}^{2}{ }^{1} \mathrm{D}_{2}$ & & & & $7.330+02$ \\
\hline $2 \mathrm{~s} 2 \mathrm{p}^{3}{ }^{3} \mathrm{D}_{1}^{o}$ & $2 \mathrm{~s}^{2} 2 \mathrm{p}^{2}{ }^{1} \mathrm{D}_{2}$ & & & & $5.928+02$ \\
\hline & $2 \mathrm{~s}^{2} 2 \mathrm{p}^{2}{ }^{1} \mathrm{D}_{2}$ & & & & $2.297+03$ \\
\hline $2 \mathrm{~s} 2 \mathrm{p}^{3}{ }^{3} \mathrm{P}_{1}^{o}$ & $2 \mathrm{~s}^{2} 2 \mathrm{p}^{2}{ }^{1} \mathrm{D}_{2}$ & 93900.40 & $5.41+03$ & $5.87+03$ & $5.158+03$ \\
\hline $2 \mathrm{~s} 2 \mathrm{p}^{3}{ }^{1} \mathrm{D}_{2}^{o}$ & $2 \mathrm{~s}^{2} 2 \mathrm{p}^{2}{ }^{1} \mathrm{D}_{2}$ & 128871.70 & $3.25+09$ & $3.21+09$ & $3.143+09$ \\
\hline & & & & & \\
\hline & $\mathrm{S}_{0}$ & 76527.80 & $2.01+03$ & $2.71+03$ & $2.057+03$ \\
\hline
\end{tabular}

assumes that the atomic state functions are built from the same orthogonal radial orbital set. However, this restriction can be relaxed with the use of the technique developed by Malmquist [37]. In order to compute transition matrix elements between two atomic state functions described by two independently optimized orbital sets, the wavefunction representations of the two states must undergo a biorthogonal transformation. The transformation of the atomic state functions is performed in such a way that the orbital sets become mutually orthogonal (biorthogonal), in which case the calculation of transition matrix elements can be handled using standard Racah techniques [14].

\section{Generation of configuration expansions}

The calculations were done term by term for all terms belonging to the $2 s^{2} 2 p^{2}$ and $2 s 2 p^{3}$ configurations. The atomic wavefunctions were generated separately for each term. Within a given term all fine structure levels were represented by wavefunctions optimized together in an EOL scheme. The fine structure levels within a term were weighted according to their statistical weights. For the terms belonging to the $2 \mathrm{~s}^{2} 2 \mathrm{p}^{2}$ ground configuration the configuration expansions were generated by single and double substitutions (SD expansion) from the $\left\{2 \mathrm{~s}^{2} 2 \mathrm{p}^{2}, 2 \mathrm{p}^{4}\right\}$ multireference set to an active set of orbitals. For the terms belonging to the $2 \mathrm{~s} 2 \mathrm{p}^{3}$ 
Table 11. Transition rates (Babushkin and Coulomb gauge) in s ${ }^{-1}$ for O III. $A_{\mathrm{MCHF}}$-from [21]; $A_{\mathrm{CIV} 3}$-from [22]; $A_{\mathrm{NIST}}$-from NIST database [41]; in the last column we quoted only the values with NIST accuracy code 'A', i.e. better than $3 \%$. Transition energies $\Delta E_{\text {exp }}$ are given in units of $\mathrm{cm}^{-1}$ and are those of [40].

\begin{tabular}{|c|c|c|c|c|c|c|c|}
\hline Upper & Lower & $\Delta E_{\exp }$ & $A_{B}$ & $A_{C}$ & $A_{\mathrm{MCHF}}$ & $A_{\mathrm{CIV} 3}$ & $A_{\mathrm{NIST}}$ \\
\hline $2 \mathrm{~s} 2 \mathrm{p}^{3{ }^{3}} \mathrm{D}_{1}^{o}$ & $2 \mathrm{~s}^{2} 2 \mathrm{p}^{2}{ }^{3} \mathrm{P}_{0}$ & 120058.20 & $3.50+08$ & $3.51+08$ & $3.489+08$ & $3.505+08$ & $3.41+08$ \\
\hline $2 \mathrm{~s} 2 \mathrm{p}^{3}{ }^{3} \mathrm{P}_{1}^{o}$ & $2 \mathrm{~s}^{2} 2 \mathrm{p}^{2}{ }^{3} \mathrm{P}_{0}$ & 142381.80 & $6.11+08$ & $6.07+08$ & $6.112+08$ & $6.595+08$ & $6.06+08$ \\
\hline $2 \mathrm{~s} 2 \mathrm{p}^{3}{ }^{3} \mathrm{~S}_{1}^{o}$ & $2 \mathrm{~s}^{2} 2 \mathrm{p}^{2}{ }^{3} \mathrm{P}_{0}$ & 197087.70 & $1.59+09$ & $1.58+09$ & $1.588+09$ & $1.718+09$ & $1.61+09$ \\
\hline $2 \mathrm{~s} 2 \mathrm{p}^{3}{ }^{1} \mathrm{P}_{1}^{o}$ & $2 \mathrm{~s}^{2} 2 \mathrm{p}^{2}{ }^{3} \mathrm{P}_{0}$ & 210461.80 & $5.09+04$ & $5.25+04$ & $6.279+04$ & $2.400+04$ & \\
\hline $2 \mathrm{~s} 2 \mathrm{p}^{35} \mathrm{~S}_{2}^{o}$ & $2 \mathrm{~s}^{2} 2 \mathrm{p}^{2}{ }^{3} \mathrm{P}_{1}$ & 60211.60 & $2.15+02$ & $3.79+02$ & $2.308+02$ & $1.668+02$ & \\
\hline $2 \mathrm{~s} 2 \mathrm{p}^{3}{ }^{3} \mathrm{D}_{2}^{o}$ & $2 \mathrm{~s}^{2} 2 \mathrm{p}^{2}{ }^{3} \mathrm{P}_{1}$ & 119940.20 & $4.71+08$ & $4.73+08$ & $4.693+08$ & $4.716+08$ & $4.58+08$ \\
\hline $2 \mathrm{~s} 2 \mathrm{p}^{3}{ }^{3} \mathrm{D}_{1}^{o}$ & $2 \mathrm{~s}^{2} 2 \mathrm{p}^{2}{ }^{3} \mathrm{P}_{1}$ & 119945.00 & $2.55+08$ & $2.56+08$ & $2.542+08$ & $2.564+08$ & $2.48+08$ \\
\hline $2 \mathrm{~s} 2 \mathrm{p}^{3}{ }^{3} \mathrm{P}_{2}^{o}$ & $2 \mathrm{~s}^{2} 2 \mathrm{p}^{2}{ }^{3} \mathrm{P}_{1}$ & 142267.80 & $4.50+08$ & $4.47+08$ & $4.506+08$ & $4.871+08$ & $4.47+08$ \\
\hline $2 \mathrm{~s} 2 \mathrm{p}^{3}{ }^{3} \mathrm{P}_{1}^{o}$ & $2 s^{2} 2 p^{2}{ }^{3} P_{1}$ & 142268.60 & $4.70+08$ & $4.67+08$ & $4.706+08$ & $5.060+08$ & $4.66+08$ \\
\hline $2 \mathrm{~s} 2 \mathrm{p}^{3}{ }^{3} \mathrm{P}_{0}^{o}$ & $2 s^{2} 2 p^{2}{ }^{3} P_{1}$ & 142280.30 & $1.84+09$ & $1.83+09$ & $1.845+09$ & $1.987+09$ & $1.83+09$ \\
\hline $2 \mathrm{~s} 2 \mathrm{p}^{31} \mathrm{D}_{2}^{o}$ & $2 s^{2} 2 p^{2}{ }^{3} P_{1}$ & 186940.80 & $1.26+04$ & $1.10+04$ & $1.325+04$ & $1.175+04$ & \\
\hline $2 \mathrm{~s} 2 \mathrm{p}^{3}{ }^{3} \mathrm{~S}_{1}^{o}$ & $2 s^{2} 2 p^{2}{ }^{3} P_{1}$ & 196974.50 & $4.77+09$ & $4.74+09$ & $4.765+09$ & $5.157+09$ & $4.82+09$ \\
\hline $2 \mathrm{~s} 2 \mathrm{p}^{3}{ }^{1} \mathrm{P}_{1}^{o}$ & $2 \mathrm{~s}^{2} 2 \mathrm{p}^{2}{ }^{3} \mathrm{P}_{1}$ & 210348.60 & $8.40+05$ & $8.29+05$ & $8.959+05$ & $4.937+05$ & \\
\hline $2 \mathrm{~s} 2 \mathrm{p}^{35} \mathrm{~S}_{2}^{o}$ & $2 \mathrm{~s}^{2} 2 \mathrm{p}^{2}{ }^{3} \mathrm{P}_{2}$ & .60 & 02 & -03 & $5.765+02$ & $4.148+02$ & \\
\hline $2 \mathrm{~s} 2 \mathrm{p}^{3}{ }^{3} \mathrm{D}_{3}^{o}$ & $2 \mathrm{~s}^{2} 2 \mathrm{p}^{2}{ }^{3} \mathrm{P}_{2}$ & 1197 & $6.17+08$ & $6.19+08$ & $6.138+08$ & $6.182+08$ & $5.99+08$ \\
\hline $2 \mathrm{~s} 2 \mathrm{p}^{3}{ }^{3} \mathrm{D}_{2}^{o}$ & $2 s^{2} 2 p^{2}{ }^{3} P_{2}$ & 119747.20 & $1.49+08$ & $1.49+08$ & $1.478+08$ & $1.497+08$ & $1.44+08$ \\
\hline $2 \mathrm{~s} 2 \mathrm{p}^{3}{ }^{3} \mathrm{D}_{1}^{o}$ & $2 \mathrm{~s}^{2} 2 \mathrm{p}^{2}{ }^{3} \mathrm{P}_{2}$ & 119752.00 & $1.61+07$ & $1.61+07$ & $1.603+07$ & $1.629+07$ & \\
\hline $2 \mathrm{~s} 2 \mathrm{p}^{3}{ }^{3} \mathrm{P}_{2}^{o}$ & $2 \mathrm{~s}^{2} 2 \mathrm{p}^{2}{ }^{3} \mathrm{P}_{2}$ & 142074.80 & $1.39+09$ & $1.38+09$ & $1.387+09$ & $1.493+09$ & $1.37+09$ \\
\hline $2 \mathrm{~s} 2 \mathrm{p}^{3}{ }^{3} \mathrm{P}_{1}^{o}$ & $2 \mathrm{~s}^{2} 2 \mathrm{p}^{2}{ }^{3} \mathrm{P}_{2}$ & 142075.60 & $7.60+08$ & $7.54+08$ & $7.608+08$ & $8.192+08$ & $7.54+08$ \\
\hline $2 \mathrm{~s} 2 \mathrm{p}^{31}{ }^{1} \mathrm{D}_{2}^{o}$ & $2 \mathrm{~s}^{2} 2 \mathrm{p}^{2}{ }^{3} \mathrm{P}_{2}$ & 186747.80 & $2.78+05$ & $2.65+05$ & $2.811+05$ & $1.885+05$ & \\
\hline $2 \mathrm{~s} 2 \mathrm{p}^{3}{ }^{3} \mathrm{~S}_{1}^{o}$ & $2 s^{2} 2 p^{2}{ }^{3} P_{2}$ & 196781.50 & $7.96+09$ & $7.92+09$ & $7.948+09$ & $8.603+09$ & $8.04+09$ \\
\hline $2 \mathrm{~s} 2 \mathrm{p}^{3}{ }^{1} \mathrm{P}_{1}^{o}$ & $2 \mathrm{~s}^{2} 2 \mathrm{p}^{2}{ }^{3} \mathrm{P}_{2}$ & 210155.60 & $1.81+05$ & $1.78+05$ & $1.947+05$ & $3.894+04$ & \\
\hline $2 \mathrm{~s} 2 \mathrm{p}^{35} \mathrm{~S}_{2}^{o}$ & $2 \mathrm{~s}^{2} 2 \mathrm{p}^{2}{ }^{1} \mathrm{D}_{2}$ & & 3 & 2 & 03 & 9.2 & \\
\hline $2 \mathrm{~s} 2 \mathrm{p}^{3}{ }^{3} \mathrm{D}_{3}^{o}$ & $2 \mathrm{~s}^{2} 2 \mathrm{p}^{2}{ }^{1} \mathrm{D}_{2}$ & 99751.90 & $2.01+04$ & $2.33+04$ & $2.108+04$ & $1.329+04$ & \\
\hline $2 \mathrm{~s} 2 \mathrm{p}^{3}{ }^{3} \mathrm{D}_{2}^{o}$ & $2 \mathrm{~s}^{2} 2 \mathrm{p}^{2}{ }^{1} \mathrm{D}_{2}$ & 99780.10 & $4.04+03$ & $4.80+03$ & $4.093+03$ & $2.425+03$ & \\
\hline $2 \mathrm{~s} 2 \mathrm{p}^{3}{ }^{3} \mathrm{D}_{1}^{o}$ & $2 \mathrm{~s}^{2} 2 \mathrm{p}^{2}{ }^{1} \mathrm{D}_{2}$ & 99784.90 & $2.13+03$ & $3.42+03$ & $2.358+03$ & $2.239+03$ & \\
\hline $2 \mathrm{~s} 2 \mathrm{p}^{3}{ }^{3} \mathrm{P}_{2}^{o}$ & $2 \mathrm{~s}^{2} 2 \mathrm{p}^{2}{ }^{1} \mathrm{D}_{2}$ & 122107.70 & $6.71+03$ & $5.62+03$ & $7.088+03$ & $2.524+03$ & \\
\hline $2 \mathrm{~s} 2 \mathrm{p}^{3}{ }^{3} \mathrm{P}_{1}^{o}$ & $2 \mathrm{~s}^{2} 2 \mathrm{p}^{2}{ }^{1} \mathrm{D}_{2}$ & 122108.50 & $2.72+04$ & $2.89+04$ & $2.746+04$ & $2.024+04$ & \\
\hline $2 \mathrm{~s} 2 \mathrm{p}^{3}{ }^{1} \mathrm{D}_{2}^{o}$ & $2 \mathrm{~s}^{2} 2 \mathrm{p}^{2}{ }^{1} \mathrm{D}_{2}$ & 166780.70 & $5.48+09$ & $5.45+09$ & $5.463+09$ & $5.561+09$ & $5.41+09$ \\
\hline $2 \mathrm{~s} 2 \mathrm{p}^{3}{ }^{3} \mathrm{~S}_{1}^{o}$ & $2 \mathrm{~s}^{2} 2 \mathrm{p}^{2}{ }^{1} \mathrm{D}_{2}$ & 176814.40 & $2.37+05$ & $2.29+05$ & $3.093+05$ & $1.030+05$ & \\
\hline $2 \mathrm{~s} 2 \mathrm{p}^{3}{ }^{1} \mathrm{P}_{1}^{o}$ & $2 \mathrm{~s}^{2} 2 \mathrm{p}^{2}{ }^{1} \mathrm{D}_{2}$ & 190188.50 & $9.23+09$ & $9.09+09$ & $9.380+09$ & $1.071+10$ & $9.60+09$ \\
\hline $2 \mathrm{~s} 2 \mathrm{p}^{3}{ }^{3} \mathrm{D}_{1}^{o}$ & $2 \mathrm{~s}^{2} 2 \mathrm{p}^{2}{ }^{1} \mathrm{~S}_{0}$ & 76872.50 & 1.86 & $1.65+03$ & $1.699+03$ & $8.307+02$ & \\
\hline $2 \mathrm{~s} 2 \mathrm{p}^{3}{ }^{3} \mathrm{P}_{1}^{o}$ & $2 \mathrm{~s}^{2} 2 \mathrm{p}^{2}{ }^{1} \mathrm{~S}_{0}$ & 99196.10 & $1.03+04$ & $1.15+04$ & $9.578+03$ & $6.108+03$ & \\
\hline $2 \mathrm{~s} 2 \mathrm{p}^{3}{ }^{3} \mathrm{~S}_{1}^{o}$ & $2 \mathrm{~s}^{2} 2 \mathrm{p}^{2}{ }^{1} \mathrm{~S}_{0}$ & 153902.00 & $6.56+04$ & $6.73+04$ & $6.975+04$ & $2.168+04$ & \\
\hline $2 \mathrm{~s} 2 \mathrm{p}^{31} \mathrm{P}_{1}^{o}$ & $2 \mathrm{~s}^{2} 2 \mathrm{p}^{2}{ }^{1} \mathrm{~S}_{0}$ & 167276.10 & $1.60+09$ & $1.62+09$ & $1.508+09$ & $1.500+09$ & $1.49+09$ \\
\hline
\end{tabular}

configuration the configuration lists were generated by SD substitutions from the single reference configuration to an active set of orbitals. In order to monitor the convergence of computed properties the active sets were systematically enlarged to include orbitals with principal quantum numbers $n=3 \ldots 8$, and orbital quantum numbers $l=0 \ldots 5$ (i.e. angular symmetries s, p, d, f, g, h). Due to stability problems in the self-consistent field procedure the optimization of radial orbitals was done layer by layer. The configuration expansions involved valence, core-valence and core-core electron correlation effects and should be adequate to also describe inner properties, like hyperfine structure.

The self-consistent field calculations for each layer of orbitals were followed by relativistic configuration interaction (RCI) calculations, including the Breit interaction. At the final stage the multireference set for the terms of the $2 \mathrm{~s}^{2} 2 \mathrm{p}^{2}$ configuration was enlarged to include $\left\{2 s^{2} 2 p^{2}, 2 p^{4}, 2 s 2 p^{2} 3 d, 2 s^{2} 3 d^{2}\right\}$. The multireference was chosen based on the criteria that it should contain the configurations that had the largest weights in the preceding self-consistent field calculations. Among the states generated by SD-excitations from the multireference set only those interacting with the multireference states were kept. In the same way the multireference set for $2 \mathrm{~s} 2 \mathrm{p}^{3}$ was enlarged to include the configurations $\left\{2 \mathrm{~s} 2 \mathrm{p}^{3}, 2 \mathrm{p}^{3} 3 \mathrm{~d}, 2 \mathrm{~s}^{2} 2 \mathrm{p} 3 \mathrm{~d}, 2 \mathrm{~s} 2 \mathrm{p} 3 \mathrm{~d}^{2}\right\}$. The leading QED effects-vacuum polarization and selfenergy — were added, as perturbative corrections, to the results of the final multireference RCI calculations.

The configuration space was explored in two directions in the current work: through the enlarged active set of orbitals, and through the increased multireference set. It would be desirable to increase the size of both sets further, but it would cross the limit imposed by the computational resources at hand. All calculations were performed on a single processor machine with $3 \mathrm{~Gb}$ internal memory and the largest multireference expansion, the one for the $2 \mathrm{~s} 2 \mathrm{p}^{3}{ }^{3} \mathrm{D}_{1,2,3}^{o}$ states, contained almost 900000 CSFs. 
Table 12. Transition rates (Babushkin and Coulomb gauge) in $\mathrm{s}^{-1}$ for F IV. $A_{\mathrm{MCHF}}$-from [21]; $A_{\mathrm{CIV} 3}$-from [24]; $A_{\mathrm{HFR}}$-from [26]. Transition energies $\Delta E_{\text {exp }}$ are given in units of $\mathrm{cm}^{-1}$ and are those of [40].

\begin{tabular}{|c|c|c|c|c|c|c|c|}
\hline Upper & Lower & $\Delta E_{\exp }$ & $A_{B}$ & $A_{C}$ & $A_{\mathrm{MCHF}}$ & $A_{\mathrm{CIV} 3}$ & $A_{\mathrm{HFR}}$ \\
\hline $2 \mathrm{~s} 2 \mathrm{p}^{3}{ }^{3} \mathrm{D}_{1}^{o}$ & $2 \mathrm{~s}^{2} 2 \mathrm{p}^{2}{ }^{3} \mathrm{P}_{0}$ & 147903.50 & $5.00+08$ & $5.01+08$ & $5.051+08$ & $5.045+08$ & $5.06+08$ \\
\hline $2 \mathrm{~s} 2 \mathrm{p}^{3}{ }^{3} \mathrm{P}_{1}^{o}$ & $2 \mathrm{~s}^{2} 2 \mathrm{p}^{2}{ }^{3} \mathrm{P}_{0}$ & 175241.90 & $8.18+08$ & $8.15+08$ & $8.277+08$ & $8.779+08$ & $8.84+08$ \\
\hline $2 \mathrm{~s} 2 \mathrm{p}^{3}{ }^{3} \mathrm{~S}_{1}^{o}$ & $2 \mathrm{~s}^{2} 2 \mathrm{p}^{2}{ }^{3} \mathrm{P}_{0}$ & 238296.70 & $1.99+09$ & $1.98+09$ & $2.002+09$ & $2.118+09$ & $1.87+09$ \\
\hline $2 \mathrm{~s} 2 \mathrm{p}^{3}{ }^{1} \mathrm{P}_{1}^{o}$ & $2 \mathrm{~s}^{2} 2 \mathrm{p}^{2}{ }^{3} \mathrm{P}_{0}$ & 257386.50 & $6.12+04$ & $6.42+04$ & $6.329+04$ & $3.180+04$ & \\
\hline $2 \mathrm{~s} 2 \mathrm{p}^{35} \mathrm{~S}_{2}^{o}$ & $2 \mathrm{~s}^{2} 2 \mathrm{p}^{2}{ }^{3} \mathrm{P}_{1}$ & 73968.70 & $7.78+02$ & $1.25+03$ & $8.225+02$ & $6.211+02$ & \\
\hline $2 \mathrm{~s} 2 \mathrm{p}^{3}{ }^{3} \mathrm{D}_{2}^{o}$ & $2 s^{2} 2 p^{2}{ }^{3} P_{1}$ & 147662.70 & $6.71+08$ & $6.72+08$ & $6.778+08$ & $6.773+08$ & $6.77+08$ \\
\hline $2 \mathrm{~s} 2 \mathrm{p}^{3}{ }^{3} \mathrm{D}_{1}^{o}$ & $2 \mathrm{~s}^{2} 2 \mathrm{p}^{2}{ }^{3} \mathrm{P}_{1}$ & 147677.50 & $3.59+08$ & $3.58+08$ & $3.618+08$ & $3.630+08$ & $3.58+08$ \\
\hline $2 \mathrm{~s} 2 \mathrm{p}^{3}{ }^{3} \mathrm{P}_{2}^{o}$ & $2 \mathrm{~s}^{2} 2 \mathrm{p}^{2}{ }^{3} \mathrm{P}_{1}$ & 175010.80 & $5.97+08$ & $5.95+08$ & $6.036+08$ & $6.416+08$ & $6.44+08$ \\
\hline $2 \mathrm{~s} 2 \mathrm{p}^{3}{ }^{3} \mathrm{P}_{1}^{o}$ & $2 \mathrm{~s}^{2} 2 \mathrm{p}^{2}{ }^{3} \mathrm{P}_{1}$ & 175015.90 & $6.41+08$ & $6.39+08$ & $6.486+08$ & $6.848+08$ & $6.93+08$ \\
\hline $2 \mathrm{~s} 2 \mathrm{p}^{3}{ }^{3} \mathrm{P}_{0}^{o}$ & $2 \mathrm{~s}^{2} 2 \mathrm{p}^{2}{ }^{3} \mathrm{P}_{1}$ & 175037.90 & $2.48+09$ & $2.47+09$ & $2.508+09$ & $2.654+09$ & $2.67+09$ \\
\hline $2 \mathrm{~s} 2 \mathrm{p}^{3}{ }^{1} \mathrm{D}_{2}^{o}$ & $2 \mathrm{~s}^{2} 2 \mathrm{p}^{2}{ }^{3} \mathrm{P}_{1}$ & 228677.80 & $4.38+04$ & $3.95+04$ & $4.409+04$ & $4.063+04$ & \\
\hline $2 \mathrm{~s} 2 \mathrm{p}^{3}{ }^{3} \mathrm{~S}_{1}^{o}$ & $2 \mathrm{~s}^{2} 2 \mathrm{p}^{2}{ }^{3} \mathrm{P}_{1}$ & 238070.70 & $5.96+09$ & $5.94+09$ & $6.007+09$ & $6.357+09$ & $5.65+09$ \\
\hline $2 \mathrm{~s} 2 \mathrm{p}^{3}{ }^{1} \mathrm{P}_{1}^{o}$ & $2 \mathrm{~s}^{2} 2 \mathrm{p}^{2}{ }^{3} \mathrm{P}_{1}$ & 257160.50 & $2.07+06$ & $2.04+06$ & $2.091+06$ & $1.406+06$ & \\
\hline $2 \mathrm{~s} 2 \mathrm{p}^{35} \mathrm{~S}_{2}^{o}$ & $2 \mathrm{p}^{2}{ }^{3} \mathrm{P}_{2}$ & & & 3.34 & -03 & 1.56 & \\
\hline $2 \mathrm{~s} 2 \mathrm{p}^{33} \mathrm{D}_{3}^{o}$ & $2 \mathrm{~s}^{2} 2 \mathrm{p}^{2}{ }^{3} \mathrm{P}_{2}$ & 147229.00 & $8.69+08$ & $8.71+08$ & $8.767+08$ & $8.785+08$ & $8.81+08$ \\
\hline $2 \mathrm{~s} 2 \mathrm{p}^{33} \mathrm{D}_{2}^{o}$ & $2 \mathrm{~s}^{2} 2 \mathrm{p}^{2}{ }^{3} \mathrm{P}_{2}$ & 147274.70 & $2.05+08$ & $2.05+08$ & $2.064+08$ & $2.082+08$ & $2.05+08$ \\
\hline $2 \mathrm{~s} 2 \mathrm{p}^{33} \mathrm{D}_{1}^{o}$ & $2 \mathrm{~s}^{2} 2 \mathrm{p}^{2}{ }^{3} \mathrm{P}_{2}$ & 147289.50 & $2.19+07$ & $2.19+07$ & $2.207+07$ & $2.236+07$ & $1.96+07$ \\
\hline $2 \mathrm{~s} 2 \mathrm{p}^{3}{ }^{3} \mathrm{P}_{2}^{o}$ & $2 \mathrm{~s}^{2} 2 \mathrm{p}^{2}{ }^{3} \mathrm{P}_{2}$ & 174622.80 & $1.87+09$ & $1.86+09$ & $1.889+09$ & $1.998+09$ & $2.02+09$ \\
\hline $2 \mathrm{~s} 2 \mathrm{p}^{3}{ }^{3} \mathrm{P}_{1}^{o}$ & $2 \mathrm{~s}^{2} 2 \mathrm{p}^{2}{ }^{3} \mathrm{P}_{2}$ & 174627.90 & $1.01+09$ & $1.01+09$ & $1.026+09$ & $1.086+09$ & $1.10+09$ \\
\hline $2 \mathrm{~s} 2 \mathrm{p}^{31} \mathrm{D}_{2}^{o}$ & $2 \mathrm{~s}^{2} 2 \mathrm{p}^{2}{ }^{3} \mathrm{P}_{2}$ & 228289.80 & $9.65+05$ & $9.30+05$ & $9.762+05$ & $7.162+05$ & \\
\hline $2 \mathrm{~s} 2 \mathrm{p}^{3}{ }^{3} \mathrm{~S}_{1}^{o}$ & $2 \mathrm{~s}^{2} 2 \mathrm{p}^{2}{ }^{3} \mathrm{P}_{2}$ & 237682.70 & $9.96+09$ & $9.92+09$ & $1.003+10$ & $1.062+10$ & $9.45+10$ \\
\hline $2 \mathrm{~s} 2 \mathrm{p}^{3}{ }^{1} \mathrm{P}_{1}^{o}$ & $2 \mathrm{~s}^{2} 2 \mathrm{p}^{2}{ }^{3} \mathrm{P}_{2}$ & 256772.50 & $3.10+05$ & $3.03+05$ & $3.076+05$ & $1.055+05$ & \\
\hline $2 \mathrm{~s} 2 \mathrm{p}^{35} \mathrm{~S}_{2}^{o}$ & $2 \mathrm{~s}^{2} 2 \mathrm{p}^{2}{ }^{1} \mathrm{D}_{2}$ & .50 & -02 & -01 & -02 & 1.40 & \\
\hline $2 \mathrm{~s} 2 \mathrm{p}^{3}{ }^{3} \mathrm{D}_{3}^{o}$ & $2 \mathrm{~s}^{2} 2 \mathrm{p}^{21} \mathrm{D}_{2}$ & 122604.80 & $7.31+04$ & $8.18+04$ & $7.694+04$ & $5.269+04$ & \\
\hline $2 \mathrm{~s} 2 \mathrm{p}^{33} \mathrm{D}_{2}^{o}$ & $2 \mathrm{~s}^{2} 2 \mathrm{p}^{2}{ }^{1} \mathrm{D}_{2}$ & 122650.50 & $1.51+04$ & $1.71+04$ & $1.572+04$ & $1.021+04$ & \\
\hline $2 \mathrm{~s} 2 \mathrm{p}^{33} \mathrm{D}_{1}^{o}$ & $2 \mathrm{~s}^{2} 2 \mathrm{p}^{2}{ }^{1} \mathrm{D}_{2}$ & 122665.30 & $7.74+03$ & $1.15+04$ & $8.274+03$ & $7.924+03$ & \\
\hline $2 \mathrm{~s} 2 \mathrm{p}^{3}{ }^{3} \mathrm{P}_{2}^{o}$ & $2 \mathrm{~s}^{2} 2 \mathrm{p}^{2}{ }^{1} \mathrm{D}_{2}$ & 149998.60 & $1.93+04$ & $1.67+04$ & $1.932+04$ & $8.633+03$ & \\
\hline $2 \mathrm{~s} 2 \mathrm{p}^{33} \mathrm{P}_{1}^{o}$ & $2 \mathrm{~s}^{2} 2 \mathrm{p}^{2}{ }^{1} \mathrm{D}_{2}$ & 150003.70 & $9.64+04$ & $1.01+05$ & $1.000+05$ & $7.639+04$ & \\
\hline $2 \mathrm{~s} 2 \mathrm{p}^{3}{ }^{1} \mathrm{D}_{2}^{o}$ & $2 \mathrm{~s}^{2} 2 \mathrm{p}^{2}{ }^{1} \mathrm{D}_{2}$ & 203665.60 & $7.52+09$ & $7.50+09$ & $7.581+09$ & $7.710+09$ & $7.00+09$ \\
\hline $2 \mathrm{~s} 2 \mathrm{p}^{3}{ }^{3} \mathrm{~S}_{1}^{o}$ & $2 \mathrm{~s}^{2} 2 \mathrm{p}^{21} \mathrm{D}_{2}$ & 213058.50 & $3.90+05$ & $3.80+05$ & $4.273+05$ & $1.988+05$ & \\
\hline $2 \mathrm{~s} 2 \mathrm{p}^{3}{ }^{1} \mathrm{P}_{1}^{o}$ & $2 \mathrm{~s}^{2} 2 \mathrm{p}^{2}{ }^{1} \mathrm{D}_{2}$ & 232148.30 & $1.12+10$ & $1.11+10$ & $1.132+10$ & $1.241+10$ & $1.15+10$ \\
\hline $2 \mathrm{~s} 2 \mathrm{p}^{3}{ }^{3} \mathrm{D}_{1}^{o}$ & $2 \mathrm{~s}^{2} 2 \mathrm{p}^{2}{ }^{1} \mathrm{~S}_{0}$ & 94362.30 & $5.92+03$ & 6.05 & $6.124+03$ & 3.3 & \\
\hline $2 \mathrm{~s} 2 \mathrm{p}^{3}{ }^{3} \mathrm{P}_{1}^{o}$ & $2 \mathrm{~s}^{2} 2 \mathrm{p}^{2}{ }^{1} \mathrm{~S}_{0}$ & 121700.70 & $3.32+04$ & $4.00+04$ & $3.381+04$ & $2.299+04$ & \\
\hline $2 \mathrm{~s} 2 \mathrm{p}^{33} \mathrm{~S}_{1}^{o}$ & $2 \mathrm{~s}^{2} 2 \mathrm{p}^{2}{ }^{1} \mathrm{~S}_{0}$ & 184755.50 & $1.68+05$ & $1.72+05$ & $1.688+05$ & $7.878+04$ & \\
\hline $2 \mathrm{~s} 2 \mathrm{p}^{3}{ }^{1} \mathrm{P}_{1}^{o}$ & $2 \mathrm{~s}^{2} 2 \mathrm{p}^{2}{ }^{1} \mathrm{~S}_{0}$ & 203845.30 & $2.37+09$ & $2.38+09$ & $2.354+09$ & $2.342+09$ & $1.94+09$ \\
\hline
\end{tabular}

\section{Results and evaluation of data}

Table 1 displays the experimental energy levels and the computed transition energies as functions of the increasing active sets and multireference sets for F IV. A saturation (or stabilization) of the calculated energy values in most cases occurred within the orbital sets with $n=8$. There are three main exceptions: ${ }^{5} \mathrm{~S}_{0}^{o}$ term, for which the energy relative to the ground state is still increasing; ${ }^{1} \mathrm{D}_{0}^{o}$ and ${ }^{1} \mathrm{P}_{0}^{o}$ terms, for which the energies are decreasing. Increasing the multireference set changes energies in the correct direction, towards the experimental energies. The effect of the multireference set is comparatively large for the states which were not fully converged with respect to the orbital basis. We noted the large effects of the increased multireference set on the fine structure splittings of the terms. In tables 2 and 3 computed energies and fine-structure splittings for N II, O III, F IV, Ne $\mathrm{V}$ and Ti XVII are compared with experimental values. The overall agreement between theory and experiment is good. The larger energy differences for ${ }^{5} \mathrm{~S}_{0}^{o},{ }^{1} \mathrm{D}_{0}^{o}$ and ${ }^{1} \mathrm{P}_{0}^{o}$ terms have been discussed above. With respect to the fine-structure, splittings for the $2 \mathrm{~s} 2 \mathrm{p}^{3}{ }^{3} \mathrm{P}^{o}$ term remain problematic. The fine-structure for this term is highly irregular along the sequence and it is not described very well in the present calculations. The finestructure splitting is strongly affected by the multireference set and it would be desirable to increase it further. When comparing with the energy differences reported by Tachiev and Froese Fischer [20], we see that the separations are somewhat better described in the Breit-Pauli approximation.

In table 4 magnetic hyperfine interaction constants $A_{J}$ for $2 \mathrm{~s}^{2} 2 \mathrm{p}^{2}{ }^{3} \mathrm{P}_{1,2}$ and $2 \mathrm{~s} 2 \mathrm{p}^{3}{ }^{3} \mathrm{P}_{1,2}^{o}$ in $\mathrm{F}$ IV are shown as functions of the increasing active sets and multireference sets. The hyperfine interaction constant for the $2 \mathrm{~s}^{2} 2 \mathrm{p}^{2}{ }^{3} \mathrm{P}_{1}$ state is not converged with respect to the active set nor to the multireference set. The oscillatory behaviour can be explained by strong internal cancellation, where significant digits are lost. We can qualitatively explain this in terms of the non-relativistic theory, in which the orbital and spin-dipolar contributions to the interaction constant are both large but counteracting; therefore, the total value is mainly determined 
Table 13. Transition rates (Babushkin and Coulomb gauge) in $\mathrm{s}^{-1}$ for Ne V. $A_{\mathrm{MCHF}}$-from [21]; $A_{\mathrm{CIV} 3}$-from [23]; $A_{S S}$-from [25]. Transition energies $\Delta E_{\text {exp }}$ are given in units of $\mathrm{cm}^{-1}$ and are those of [40].

\begin{tabular}{|c|c|c|c|c|c|c|c|}
\hline Jpper & wer & $\Delta E_{\exp }$ & $A_{B}$ & $A_{C}$ & $A_{\mathrm{MCHF}}$ & $A_{\mathrm{CIV} 3}$ & $A_{S S}$ \\
\hline$P_{1}^{o}$ & $\mathrm{P}_{0}$ & 5.00 & $63+08$ & $6.63+08$ & $6.741+08$ & $6.701+08$ & $7.250+08$ \\
\hline & $\mathrm{P}_{0}$ & 3.30 & $1.03+09$ & $1.03+09$ & $1.050+09$ & $.102+09$ & $1.247+09$ \\
\hline $\mathrm{s} 2 \mathrm{p}^{3}{ }^{3} \mathrm{~S}_{1}^{o}$ & $2 \mathrm{~s}^{2} 2 \mathrm{p}^{2}{ }^{3} \mathrm{P}_{0}$ & 279371.20 & $2.39+09$ & $2.38+09$ & $2.416+09$ & $.520+09$ & $2.855+09$ \\
\hline $\mathrm{P}_{1}^{o}$ & $2 s^{2} 21$ & 3038 & $62+04$ & 0.10 .04 & $.572+04$ & $998+04$ & $1.781+05$ \\
\hline 2 & $b_{1}$ & 30 & +03 & $48+03$ & $476+03$ & $1.898+03$ & $1.797+03$ \\
\hline $2 \mathrm{p}^{3}{ }^{3} \mathrm{D}_{2}^{o}$ & & 175 & $87+08$ & $88+08$ & $019+08$ & $970+08$ & $.709+08$ \\
\hline $\mathrm{s} 2 \mathrm{p}^{3}{ }^{3} \mathrm{D}_{1}^{o}$ & $2 \mathrm{~s}^{2} 2 \mathrm{p}^{2}{ }^{3} \mathrm{P}_{1}$ & 175 & $4.64+08$ & $4.64+08$ & $4.717+08$ & $4.716+08$ & $33+08$ \\
\hline & & 207 & 7.4 & +08 & 08 & 7.9 & $76+08$ \\
\hline${ }^{3}{ }^{3} \mathrm{P}_{1}^{o}$ & & 207 & 8.2 & $8.27+08$ & 08 & 8.7 & $000+09$ \\
\hline $\mathrm{s} 2 \mathrm{p}^{3}{ }^{3} \mathrm{P}_{0}^{o}$ & & 207 & & +09 & 09 & - & 804+09 \\
\hline $\mathrm{D}_{2}^{o}$ & & 270 & 1. & +05 & & 1.1 & $.404+05$ \\
\hline $\mathrm{s} 2 \mathrm{p}^{3}{ }^{3} \mathrm{~S}_{1}^{o}$ & $2 s^{2} 2$ & 278 & 7.1 & +09 & 09 & & $8.571+09$ \\
\hline $\mathrm{s} 2 \mathrm{p}^{3}{ }^{1} \mathrm{P}_{1}^{o}$ & $2 s^{2} 2$ & 303 & 06 & $4.79+06$ & 06 & +06 & $6.929+06$ \\
\hline & & & & & & & +03 \\
\hline $\mathrm{D}_{3}^{o}$ & & 172 & & 09 & & 09 & $5+09$ \\
\hline $\mathrm{s} 2 \mathrm{p}^{3}{ }^{3} \mathrm{D}_{2}^{o}$ & $\mathrm{~s}^{2} 2 \mathrm{p}^{2}{ }^{3} \mathrm{P}_{2}$ & 174 & 08 & 08 & 08 & 08 & $4+08$ \\
\hline $\mathrm{s} 2 \mathrm{p}^{3{ }^{3}} \mathrm{D}_{1}^{o}$ & ${ }^{3} \mathrm{P}_{2}$ & & & & & & $4+07$ \\
\hline $\mathrm{s} 2 \mathrm{p}^{3}{ }^{3} \mathrm{P}_{2}^{o}$ & $2 s^{2} 2$ & & & & & & +09 \\
\hline $\mathrm{s} 2 \mathrm{p}^{3}{ }^{3} \mathrm{P}_{1}^{o}$ & $2 \mathrm{~s}^{2} 2 \mathrm{p}^{2}{ }^{3} \mathrm{P}_{2}$ & $20^{\prime}$ & & 09 & & & +09 \\
\hline $\mathrm{s} 2 \mathrm{p}^{3{ }^{1}} \mathrm{D}_{2}^{o}$ & $2 \mathrm{~s}^{2} 2 \mathrm{p}^{2}{ }^{3} \mathrm{P}_{2}$ & & & & & & +06 \\
\hline $\mathrm{s} 2 \mathrm{p}^{3}{ }^{3} \mathrm{~S}_{1}^{o}$ & $2 \mathrm{~s}^{2} 2 \mathrm{p}^{2}{ }^{3} \mathrm{P}_{2}$ & 27 & & & & & +10 \\
\hline $\mathrm{s} 2 \mathrm{p}^{3}{ }^{1} \mathrm{P}_{1}^{o}$ & $2 \mathrm{~s}^{2} 2 \mathrm{p}^{2}{ }^{3} \mathrm{P}_{2}$ & 3027 & 5.30 & 5.1 & & 2.1 & 1.2 \\
\hline $\mathrm{s} 2 \mathrm{p}^{35} \mathrm{~S}_{2}^{o}$ & $\mathrm{D}_{2}$ & & & & & & -01 \\
\hline $\mathrm{s} 2 \mathrm{p}^{3{ }^{3}} \mathrm{D}_{3}^{o}$ & ${ }^{1} \mathrm{D}_{2}$ & & & & & & +05 \\
\hline${ }^{2} \mathrm{~s} 2 \mathrm{p}^{3}{ }^{3} \mathrm{D}_{2}^{o}$ & $2 \mathrm{~s}^{2} 2 \mathrm{p}^{21} \mathrm{D}_{2}$ & & & 04 & & & +04 \\
\hline $2 \mathrm{~s} 2 \mathrm{p}^{3}{ }^{3} \mathrm{D}_{1}^{o}$ & $2 \mathrm{~s}^{2} 2 \mathrm{p}^{2}{ }^{1} \mathrm{D}_{2}$ & & 2.3 & & & & +04 \\
\hline $\mathrm{s} 2 \mathrm{p}^{3}{ }^{3} \mathrm{P}_{2}^{o}$ & $2 \mathrm{~s}^{2} 2 \mathrm{p}^{2}{ }^{1} \mathrm{D}_{2}$ & 1778 & & & & & $5+04$ \\
\hline $2 \mathrm{~s} 2 \mathrm{p}^{3}{ }^{3} \mathrm{P}_{1}^{o}$ & $2 \mathrm{~s}^{2} 2 \mathrm{p}^{2}{ }^{1} \mathrm{D}_{2}$ & 177862.60 & & & & & $3.027+05$ \\
\hline $2 \mathrm{~s} 2 \mathrm{p}^{3}{ }^{1} \mathrm{D}_{2}^{o}$ & $2 \mathrm{~s}^{2} 2 \mathrm{p}^{21} \mathrm{D}_{2}$ & 240262.20 & $9.52+09$ & $9.50+09$ & $9.657+09$ & 9.77 & $1.170+10$ \\
\hline $2 \mathrm{~s} 2 \mathrm{p}^{33} \mathrm{~S}_{1}^{o}$ & $2 \mathrm{~s}^{2} 2 \mathrm{p}^{2}{ }^{1} \mathrm{D}_{2}$ & 249080.50 & $6.66+05$ & & & & $2.190+06$ \\
\hline $2 \mathrm{~s} 2 \mathrm{p}^{3}{ }^{1} \mathrm{P}_{1}^{o}$ & $2 \mathrm{~s}^{2} 2 \mathrm{p}^{2}{ }^{1} \mathrm{D}_{2}$ & 273528.50 & $1.33+10$ & $1.33+10$ & $1.355+10$ & $1.455+10$ & $1.732+10$ \\
\hline $\mathrm{D}_{1}^{o}$ & No & & & & & & \\
\hline & $2 \mathrm{~s}^{2} 2 \mathrm{p}^{21} \mathrm{~S}_{0}$ & 144237.90 & $9.52+04$ & & & & +05 \\
\hline $2 \mathrm{~s} 2 \mathrm{p}^{3}{ }^{3} \mathrm{~S}_{1}^{o}$ & $2 \mathrm{~s}^{2} 2 \mathrm{p}^{2}{ }^{1} \mathrm{~S}_{0}$ & 2154 & & & $3.928+$ & & $5.285+05$ \\
\hline $2 \mathrm{~s} 2 \mathrm{p}^{3}{ }^{1} \mathrm{P}_{1}^{o}$ & $2 \mathrm{~s}^{2} 2 \mathrm{p}^{21} \mathrm{~S}_{0}$ & 239903.80 & $3.06+09$ & $3.07+09$ & $3.091+09$ & $3.057+09$ & $3.581+09$ \\
\hline
\end{tabular}

by the Fermi contact term. The contact term, in turn, is a sum of large and counteracting spin-polarization contributions from the $1 \mathrm{~s}$ and $2 \mathrm{~s}$ sub-shells. As a result the final value is very sensitive to correlation effects [38]. In contrast, the interaction constants for the other states are very stable and they are expected to be accurate to within a few parts in a thousand.

In table 5 magnetic dipole and electric quadrupole hyperfine interaction constants are displayed together with the Landé $g_{J}$-factors for all ions. The hyperfine shifts for the states belonging to the $2 \mathrm{~s} 2 \mathrm{p}^{3}$ configuration are dominated by large magnetic dipole interaction constants. For the $2 s^{2} 2 p^{2}$ states the electric quadrupole interaction constants are also important. The Landé $g_{J}$-factors are relatively insensitive to correlation effects. For light elements the values are close to what is expected from pure $L S$ coupling. Only for $\mathrm{Ti}$ XVII there is a considerable deviation from the $L S$ values. To the knowledge of the authors there are no experimental or theoretical data for the hyperfine interaction constants in the literature to compare with.
As noted by Clark [39] the $2 \mathrm{~s}^{2} 2 \mathrm{p}^{n}-2 \mathrm{~s} 2 \mathrm{p}^{n+1}$ transition arrays have large isotope shifts. This is due to the fact that both the normal and specific isotope shifts act in the same direction. In addition there is only a small cancellation for the specific mass shift parameters of the upper and lower state and difference $\Delta S_{\text {sms }}$ remains comparatively large. In table 6 we display specific mass shift parameters $S_{\text {sms }}$ and electron densities at the nucleus $\rho(0)$ for $2 \mathrm{~s}^{2} 2 \mathrm{p}^{2}{ }^{3} \mathrm{P}_{0}$ and $2 \mathrm{~s} 2 \mathrm{p}^{3}{ }^{3} \mathrm{P}_{0}^{o}$ in $\mathrm{F}$ IV as functions of the increasing active sets and multireference sets. Also the differences $\Delta S_{\text {sms }}$ and $\Delta \rho(0)$ are shown. The computed quantities, especially the differences, saturate quickly and there is excellent convergence. In table 7 the specific mass shift parameters and electron densities are shown for all ions. Most notable is perhaps how the specific mass shift parameters for $2 \mathrm{~s} 2 \mathrm{p}^{3}{ }^{3} \mathrm{P}^{o}$ and $2 \mathrm{~s} 2 \mathrm{p}^{3}{ }^{1} \mathrm{P}^{o}$ approach each other for large $Z$. For all ions except Ti there are only small effects of $J$, and the specific mass shifts are well described by term values. The elements $\mathrm{N}, \mathrm{O}$ and $\mathrm{Ne}$ all have more than one stable isotope. Using the data in table 7 together with experimental values for the energy separations, transition isotope shifts for 
Table 14. Transition rates (Babushkin and Coulomb gauge) in $\mathrm{s}^{-1}$ for Ti XVII. $A_{\mathrm{HFR}}$-from [26]; $A_{\mathrm{NIST}}$-from NIST [41]. 'Code' is the accuracy code associated with the NIST data: $\mathrm{C}<25 \%, \mathrm{D}<50 \%, \mathrm{E}>50 \%$. Transition energies $\Delta E_{\exp }$ are given in units of $\mathrm{cm}^{-1}$ and are those of [40].

\begin{tabular}{|c|c|c|c|c|c|c|c|}
\hline Upper & Lower & $\Delta E_{\exp }$ & $A_{B}$ & $A_{C}$ & $A_{\mathrm{HFR}}$ & $A_{\mathrm{NIST}}$ & Code \\
\hline $2 \mathrm{~s} 2 \mathrm{p}^{3}{ }^{3} \mathrm{D}_{1}^{o}$ & $2 \mathrm{~s}^{2} 2 \mathrm{p}^{2}{ }^{3} \mathrm{P}_{0}$ & 580110.00 & $5.80+09$ & $5.79+09$ & $5.67+09$ & $6.4+09$ & $\mathrm{C}$ \\
\hline $2 \mathrm{~s} 2 \mathrm{p}^{3}{ }^{3} \mathrm{P}_{1}^{o}$ & $2 \mathrm{~s}^{2} 2 \mathrm{p}^{2}{ }^{3} \mathrm{P}_{0}$ & 680910.00 & $3.59+09$ & $3.60+09$ & $3.61+09$ & $3.79+09$ & $\mathrm{C}$ \\
\hline $2 \mathrm{~s} 2 \mathrm{p}^{3}{ }^{3} \mathrm{~S}_{1}^{o}$ & $2 \mathrm{~s}^{2} 2 \mathrm{p}^{2}{ }^{3} \mathrm{P}_{0}$ & 838340.00 & $7.61+09$ & $7.60+09$ & $7.34+09$ & $8.0+09$ & $\mathrm{C}$ \\
\hline $2 \mathrm{~s} 2 \mathrm{p}^{3}{ }^{1} \mathrm{P}_{1}^{o}$ & $2 \mathrm{~s}^{2} 2 \mathrm{p}^{2}{ }^{3} \mathrm{P}_{0}$ & 943500.00 & $1.07+07$ & $1.11+07$ & & & \\
\hline $2 \mathrm{~s} 2 \mathrm{p}^{35} \mathrm{~S}_{2}^{o}$ & $2 \mathrm{~s}^{2} 2 \mathrm{p}^{2}{ }^{3} \mathrm{P}_{1}$ & 304002.00 & $5.73+06$ & $6.51+06$ & & $6.6+06$ & $\mathrm{E}$ \\
\hline $2 \mathrm{~s} 2 \mathrm{p}^{3}{ }^{3} \mathrm{D}_{2}^{o}$ & $2 s^{2} 2 p^{2}{ }^{3} P_{1}$ & 549232.00 & $6.00+09$ & $6.02+09$ & $5.91+09$ & $6.6+09$ & $\mathrm{D}$ \\
\hline $2 \mathrm{~s} 2 \mathrm{p}^{3}{ }^{3} \mathrm{D}_{1}^{o}$ & $2 s^{2} 2 p^{2}{ }^{3} P_{1}$ & 550452.00 & $1.19+09$ & $1.18+09$ & $1.14+09$ & $1.3+09$ & $\mathrm{D}$ \\
\hline $2 \mathrm{~s} 2 \mathrm{p}^{3}{ }^{3} \mathrm{P}_{2}^{o}$ & $2 s^{2} 2 p^{2}{ }^{3} P_{1}$ & 657122.00 & $9.96+08$ & $1.00+09$ & $9.81+08$ & $1.0+09$ & $\mathrm{D}$ \\
\hline $2 \mathrm{~s} 2 \mathrm{p}^{3}{ }^{3} \mathrm{P}_{1}^{o}$ & $2 s^{2} 2 p^{2}{ }^{3} P_{1}$ & 651252.00 & $7.74+10$ & $7.74+10$ & $7.64+10$ & $8.5+10$ & $\mathrm{C}$ \\
\hline $2 \mathrm{~s} 2 \mathrm{p}^{3}{ }^{3} \mathrm{P}_{0}^{o}$ & $2 s^{2} 2 p^{2}{ }^{3} P_{1}$ & 648792.00 & $1.47+10$ & $1.46+10$ & $1.46+10$ & $1.63+10$ & $\mathrm{C}$ \\
\hline $2 \mathrm{~s} 2 \mathrm{p}^{31}{ }^{1} \mathrm{D}_{2}^{o}$ & $2 s^{2} 2 p^{2}{ }^{3} P_{1}$ & 815482.00 & $1.20+08$ & $1.18+08$ & $8.88+07$ & $1.3+08$ & $\mathrm{E}$ \\
\hline $2 \mathrm{~s} 2 \mathrm{p}^{3}{ }^{3} \mathrm{~S}_{1}^{o}$ & $2 s^{2} 2 p^{2}{ }^{3} P_{1}$ & 808682.00 & $2.16+10$ & $2.16+10$ & $2.11+10$ & $2.3+10$ & $\mathrm{E}$ \\
\hline $2 \mathrm{~s} 2 \mathrm{p}^{3}{ }^{1} \mathrm{P}_{1}^{o}$ & $2 s^{2} 2 p^{2}{ }^{3} P_{1}$ & 913842.00 & $1.97+09$ & $1.96+09$ & $1.86+09$ & $2.2+09$ & $\mathrm{E}$ \\
\hline $2 \mathrm{~s} 2 \mathrm{p}^{35} \mathrm{~S}_{2}^{o}$ & $2 \mathrm{~s}^{2} 2 \mathrm{p}^{2}{ }^{3} \mathrm{P}_{2}$ & 277930.00 & $7.73+06$ & $9.02+06$ & & $8.3+06$ & $\mathrm{E}$ \\
\hline $2 \mathrm{~s} 2 \mathrm{p}^{3}{ }^{3} \mathrm{D}_{3}^{o}$ & $2 s^{2} 2 p^{2}{ }^{3} P_{2}$ & 531030.00 & $4.76+09$ & $4.79+09$ & $4.66+09$ & $5.2+09$ & $\mathrm{C}$ \\
\hline $2 \mathrm{~s} 2 \mathrm{p}^{3}{ }^{3} \mathrm{D}_{2}^{o}$ & $2 \mathrm{~s}^{2} 2 \mathrm{p}^{2}{ }^{3} \mathrm{P}_{2}$ & 523160.00 & $1.02+08$ & $9.98+07$ & $7.27+07$ & $8.8+07$ & $\mathrm{E}$ \\
\hline $2 \mathrm{~s} 2 \mathrm{p}^{3}{ }^{3} \mathrm{D}_{1}^{o}$ & $2 s^{2} 2 p^{2}{ }^{3} P_{2}$ & 524380.00 & $2.03+07$ & $2.11+07$ & & $1.5+07$ & $\mathrm{E}$ \\
\hline $2 \mathrm{~s} 2 \mathrm{p}^{3}{ }^{3} \mathrm{P}_{2}^{o}$ & $2 s^{2} 2 p^{2}{ }^{3} P_{2}$ & 631050.00 & $1.24+10$ & $1.24+10$ & $1.22+10$ & $1.4+10$ & $\mathrm{C}$ \\
\hline $2 \mathrm{~s} 2 \mathrm{p}^{3}{ }^{3} \mathrm{P}_{1}^{o}$ & $2 s^{2} 2 p^{2}{ }^{3} P_{2}$ & 625180.00 & $3.16+09$ & $3.14+09$ & $3.12+09$ & $3.7+09$ & $\mathrm{D}$ \\
\hline $2 \mathrm{~s} 2 \mathrm{p}^{3}{ }^{1} \mathrm{D}_{2}^{o}$ & $2 \mathrm{~s}^{2} 2 \mathrm{p}^{2}{ }^{3} \mathrm{P}_{2}$ & 789410.00 & $2.73+09$ & $2.72+09$ & $2.82+09$ & $3.0+09$ & $\mathrm{E}$ \\
\hline $2 \mathrm{~s} 2 \mathrm{p}^{3}{ }^{3} \mathrm{~S}_{1}^{o}$ & $2 s^{2} 2 p^{2}{ }^{3} P_{2}$ & 782610.00 & $4.38+10$ & $4.39+10$ & $4.28+10$ & $4.6+10$ & $\mathrm{C}$ \\
\hline $2 \mathrm{~s} 2 \mathrm{p}^{3}{ }^{1} \mathrm{P}_{1}^{o}$ & $2 \mathrm{~s}^{2} 2 \mathrm{p}^{2}{ }^{3} \mathrm{P}_{2}$ & 887770.00 & $1.38+07$ & $1.41+07$ & & & \\
\hline $2 \mathrm{~s} 2 \mathrm{p}^{35} \mathrm{~S}_{2}^{o}$ & $2 \mathrm{~s}^{2} 2 \mathrm{p}^{2}{ }^{1} \mathrm{D}_{2}$ & 193000.00 & $51+05$ & $1.94+05$ & & & \\
\hline $2 \mathrm{~s} 2 \mathrm{p}^{3}{ }^{3} \mathrm{D}_{3}^{o}$ & $2 s^{2} 2 p^{2}{ }^{1} D_{2}$ & 446100.00 & $3.01+08$ & $3.12+08$ & $3.27+08$ & $3.2+08$ & $\mathrm{E}$ \\
\hline $2 \mathrm{~s} 2 \mathrm{p}^{3}{ }^{3} \mathrm{D}_{2}^{o}$ & $2 \mathrm{~s}^{2} 2 \mathrm{p}^{2}{ }^{1} \mathrm{D}_{2}$ & 438230.00 & $2.49+07$ & $2.62+07$ & $2.59+07$ & $3.6+07$ & $\mathrm{E}$ \\
\hline $2 \mathrm{~s} 2 \mathrm{p}^{3}{ }^{3} \mathrm{D}_{1}^{o}$ & $2 s^{2} 2 p^{2}{ }^{1} D_{2}$ & 439450.00 & $5.46+07$ & $5.92+07$ & & $6.2+07$ & $\mathrm{E}$ \\
\hline $2 \mathrm{~s} 2 \mathrm{p}^{3}{ }^{3} \mathrm{P}_{2}^{o}$ & $2 \mathrm{~s}^{2} 2 \mathrm{p}^{2}{ }^{1} \mathrm{D}_{2}$ & 546120.00 & $7.16+07$ & $6.94+07$ & $8.02+07$ & $1.1+08$ & $\mathrm{E}$ \\
\hline $2 \mathrm{~s} 2 \mathrm{p}^{3}{ }^{3} \mathrm{P}_{1}^{o}$ & $2 \mathrm{~s}^{2} 2 \mathrm{p}^{2}{ }^{1} \mathrm{D}_{2}$ & 540250.00 & $1.73+08$ & $1.72+08$ & $1.96+08$ & $1.9+08$ & $\mathrm{E}$ \\
\hline $2 \mathrm{~s} 2 \mathrm{p}^{3}{ }^{1} \mathrm{D}_{2}^{o}$ & $2 \mathrm{~s}^{2} 2 \mathrm{p}^{2}{ }^{1} \mathrm{D}_{2}$ & 704480.00 & $3.59+10$ & $3.60+10$ & $3.52+10$ & $3.87+10$ & $\mathrm{C}$ \\
\hline $2 \mathrm{~s} 2 \mathrm{p}^{3}{ }^{3} \mathrm{~S}_{1}^{o}$ & $2 s^{2} 2 p^{21} D_{2}$ & 697680.00 & $1.59+07$ & $1.64+07$ & & & \\
\hline $2 \mathrm{~s} 2 \mathrm{p}^{3}{ }^{1} \mathrm{P}_{1}^{o}$ & $2 \mathrm{~s}^{2} 2 \mathrm{p}^{2}{ }^{1} \mathrm{D}_{2}$ & 802840.00 & $4.74+10$ & $4.74+10$ & $4.63+10$ & $5.2+10$ & $\mathrm{C}$ \\
\hline $2 \mathrm{~s} 2 \mathrm{p}^{33} \mathrm{D}_{1}^{o}$ & $2 \mathrm{~s}^{2} 2 \mathrm{p}^{2}{ }^{1} \mathrm{~S}_{0}$ & 337930.00 & $1.61+07$ & 1.63 & & $2.2+07$ & $\mathrm{E}$ \\
\hline $2 \mathrm{~s} 2 \mathrm{p}^{3}{ }^{3} \mathrm{P}_{1}^{o}$ & $2 \mathrm{~s}^{2} 2 \mathrm{p}^{2}{ }^{1} \mathrm{~S}_{0}$ & 438730.00 & $5.84+07$ & $6.24+07$ & $4.27+07$ & $6.8+07$ & $\mathrm{E}$ \\
\hline $2 \mathrm{~s} 2 \mathrm{p}^{3}{ }^{3} \mathrm{~S}_{1}^{o}$ & $2 \mathrm{~s}^{2} 2 \mathrm{p}^{2}{ }^{1} \mathrm{~S}_{0}$ & 596160.00 & $2.16+08$ & $2.20+08$ & $2.36+08$ & $2.2+08$ & $\mathrm{E}$ \\
\hline $2 \mathrm{~s} 2 \mathrm{p}^{3}{ }^{1} \mathrm{P}_{1}^{o}$ & $2 \mathrm{~s}^{2} 2 \mathrm{p}^{2}{ }^{1} \mathrm{~S}_{0}$ & 701320.00 & $1.26+10$ & $1.26+10$ & $1.21+10$ & $1.35+10$ & $\mathrm{C}$ \\
\hline
\end{tabular}

the different isotopic pairs have been calculated for all $E 1$ transitions in the $2 \mathrm{~s}^{2} 2 \mathrm{p}^{2}-2 \mathrm{~s} 2 \mathrm{p}^{3}$ transition array. The transition isotope shifts are displayed in table 8 . A positive sign of the transition isotope shift corresponds to a larger energy transition (i.e. a blue shifted line) for the heavier isotope. These data together with hyperfine interaction constants allow line profiles in high-resolution spectra to be modelled in detail [19].

In table 9 the transition rates for $2 \mathrm{~s}^{2} 2 \mathrm{p}^{2}{ }^{3} \mathrm{P}_{0}-2 \mathrm{~s} 2 \mathrm{p}^{3}{ }^{3} \mathrm{P}_{1}^{o}$ and $2 \mathrm{~s}^{2} 2 \mathrm{p}^{2}{ }^{1} \mathrm{D}_{2}-2 \mathrm{~s} 2 \mathrm{p}^{3}{ }^{1} \mathrm{P}_{1}^{o}$ in F IV are shown as functions of increasing active sets and multireference sets. Whereas the values for the $2 \mathrm{~s}^{2} 2 \mathrm{p}^{2}{ }^{1} \mathrm{D}_{2}-2 \mathrm{~s} 2 \mathrm{p}^{3}{ }^{1} \mathrm{P}_{1}^{o}$ transition are very stable, the values for $2 \mathrm{~s}^{2} 2 \mathrm{p}^{2}{ }^{3} \mathrm{P}_{0}-2 \mathrm{~s} 2 \mathrm{p}^{3}{ }^{3} \mathrm{P}_{1}^{o}$ oscillate at the $1 \%$ level and, again, it would be desirable to increase the orbital basis further. Rates computed in two gauges, Babushkin and Coulomb, are brought in close agreement when the multireference set is increased. Rates for all $E 1$ transitions in the $2 s^{2} 2 p^{2}-2 s 2 p^{3}$ transition array are given in tables 1014. The values from recent MCHF and CIV3 Breit-Pauli [21-24] calculations are included in the tables for N II, O III,
F IV and Ne V for comparison. For F IV and Ti XVII we also display transition rates computed by the Hartree-FockRelativistic (HFR) program [26]. In table 13 values from SUPERSTRUCTURE calculations by Bhatia and Doschek [25] are added.

Rates for almost all transitions in the $2 s^{2} 2 p^{2}-2 s 2 p^{3}$ array are also listed in the NIST database [41], but their accuracy classification renders them rather insufficient for the purpose of comparison with Breit-Pauli or current Dirac-Hartree-Fock results. The only exception is the O III, for which several entries are listed in [41] with NIST accuracy classification 'A' (i.e. better than 3\%). We included these class ' $A$ ' data in the last column of table 11 . With the exception of the two lines $2 \mathrm{~s}^{2} 2 \mathrm{p}^{2}{ }^{1} \mathrm{D}_{2}-2 \mathrm{~s} 2 \mathrm{p}^{3}{ }^{1} \mathrm{P}_{1}^{o}$ and $2 \mathrm{~s}^{2} 2 \mathrm{p}^{2}{ }^{1} \mathrm{~S}_{0}-2 \mathrm{~s} 2 \mathrm{p}^{3}{ }^{1} \mathrm{P}_{1}^{o}$ the Dirac-Hartree-Fock results are in agreement with the NIST data within their specified error bounds. For Ti XVII, table 14 we added values from the NIST database [41] together with the accuracy classifications. 
Table 15. Transition probabilities in $\mathrm{s}^{-1}$ for the $2 \mathrm{~s}^{2} 2 \mathrm{p}^{2}{ }^{3} \mathrm{P}_{J}-2 \mathrm{~s} 2 \mathrm{p}^{35} \mathrm{~S}_{2}^{o}$ intercombination lines in N II and O III, and lifetime $\tau$ in ms for the ${ }^{5} \mathrm{~S}_{2}^{o}$ level. $B R$ is the branching ratio.

\begin{tabular}{|c|c|c|c|c|}
\hline \multicolumn{2}{|c|}{$A$ values $\left(\mathrm{s}^{-1}\right)$} & \multirow[b]{2}{*}{$B R$} & \multirow[b]{2}{*}{$\tau(\mathrm{ms})$} & \multirow[b]{2}{*}{ Source } \\
\hline${ }^{5} \mathrm{~S}_{2}^{o}-{ }^{3} \mathrm{P}_{1}$ & ${ }^{5} \mathrm{~S}_{2}^{o}-{ }^{3} \mathrm{P}_{2}$ & & & \\
\hline \multicolumn{5}{|c|}{ N II } \\
\hline \multicolumn{5}{|l|}{ Theory } \\
\hline 44.7 & 110 & 2.46 & 6.46 & RCI this work \\
\hline 51.55 & 126.6 & 2.456 & 5.61 & MCHF-BP adjusted (2004) [21] \\
\hline 53.6 & 130.6 & 2.44 & 5.43 & CIV3 adjusted (1997) [45] \\
\hline \multicolumn{5}{|c|}{ Experiment } \\
\hline & & & $5.88(3)$ & Ion-trap (1998) [50] \\
\hline \multicolumn{5}{|c|}{ Observation } \\
\hline & & $2.31(40)$ & & Solar (1977) [51] \\
\hline & & $2.45(4)$ & & $\operatorname{Arc}(1996)[52]$ \\
\hline & & $2.27(23)$ & & ICP (1996) [53] \\
\hline & & $2.24(6)$ & & Arc (1996) [54] \\
\hline \multicolumn{5}{|c|}{ O III } \\
\hline \multicolumn{5}{|l|}{ Theory } \\
\hline 215 & 535 & 2.49 & 1.33 & RCI this work \\
\hline 230.8 & 576.5 & 2.498 & 1.239 & MCHF-BP adjusted (2004) [21] \\
\hline 218 & 541 & 2.482 & 1.32 & MCDF (1997) [46] \\
\hline 237 & 589 & 2.486 & 1.21 & CIV3 adjusted (1997) [46] \\
\hline 166.8 & 414.8 & 2.487 & 1.719 & CIV3 (1997) [22] \\
\hline \multicolumn{5}{|c|}{ Experiment } \\
\hline & & & $\begin{array}{l}1.22(8) \\
1.250(13)\end{array}$ & $\begin{array}{l}\text { Ion-trap (1984) [5J] } \\
\text { Ion-trap (2000) }\end{array}$ \\
\hline \multicolumn{5}{|c|}{ Observation } \\
\hline & & 2.57 & & Solar (1996) [57] \\
\hline & & $2.79(3)$ & & RR Tel(GHRS) (1996) [58] \\
\hline
\end{tabular}

The agreement between the transition rates obtained in the Babushkin and Coulomb gauges is very good for strong transitions. In weak transitions the agreement between the gauges depends on a particular term under consideration. For some transitions the agreement is good but for others, e.g. the $2 \mathrm{~s}^{2} 2 \mathrm{p}^{2}{ }^{3} P_{2}-2 \mathrm{~s} 2 \mathrm{p}^{3}{ }^{5} S_{2}^{o}$, there are substantial differences. The weakness of a transition frequently comes out as a result of cancellation between a number of large contributions or between different parts of the radial transition integrals [42]. A small unbalance due to correlation effects may thus change the calculated transition probabilities dramatically in one of the gauges. In weak transition there may be sizable effects from the negative energy states, not accounted for in the present calculation, which affect rates in the Coulomb gauge [43], and the general wisdom is that values in the Babushkin gauge should be used in such cases. The agreement between the present values and the Breit-Pauli values by Froese Fischer and Tachiev [21] is very good, especially for strong transitions. For $\mathrm{Ne} \mathrm{V}$ the rates of strong transitions obtained from the fully relativistic Dirac-Hartree-Fock calculation are consistently smaller than the ones from the Breit-Pauli calculation. This might be due to the relativistic contraction effects which are better captured in the former calculation. The calculations by Aggarwal et al [22-24] are comparatively small in terms of electron correlation effects included. Nevertheless, the general agreement between these calculations and the BreitPauli calculations by Froese Fischer and Tachiev as well as the present fully relativistic one is very satisfactory. There is a fair agreement between the SUPERSTRUCTURE calculations by Bhatia and Doschek [25] and the present calculations for $\mathrm{Ne}$ V. For Ti XVII the present values, which should be correct to within a few percent, are considerably more accurate than the values in the NIST compilation. The latter are mainly from the pioneering Dirac-Hartree-Fock calculations by Cheng et al [44]. The strongest transitions in Ti XVII have been calculated by Fawcett using the Hartree-Fock relativistic (HFR) method [26]. Although small, these calculations agree very well with the present calculation.

The $2 \mathrm{~s}^{2} 2 \mathrm{p}^{2}{ }^{3} \mathrm{P}_{J}-2 \mathrm{~s} 2 \mathrm{p}^{3}{ }^{5} \mathrm{~S}_{2}^{o}$ transition is of considerable interest in astrophysical applications (see [45] and references therein). In table 15 we compare decay rates from $2 \mathrm{~s} 2 \mathrm{p}^{3}{ }^{5} \mathrm{~S}_{2}^{o}$ to the two lower states $2 \mathrm{~s}^{2} 2 \mathrm{p}^{23} \mathrm{P}_{J}$ as well as lifetimes $\tau$ of the ${ }^{5} \mathrm{~S}_{2}^{o}$ states in $\mathrm{N}$ II and $\mathrm{O}$ III. In addition the branching ratio

$$
B R=\frac{A\left({ }^{5} \mathrm{~S}_{2}^{o}-{ }^{3} \mathrm{P}_{2}\right)}{A\left({ }^{5} \mathrm{~S}_{2}^{o}-{ }^{3} \mathrm{P}_{1}\right)},
$$

which is a measurable quantity, is given. The transition rates from the present calculations are somewhat smaller, especially for N II, than the ones from adjusted Breit-Pauli calculations [21, 45, 46], leading to a larger lifetime, as compared to accurate ion-trap measurements. All calculations give essentially the same value of the branching ratio $B R$. Considering weak intercombination transitions in general, the large cancellation of different contributions reduces the 
accuracy of the calculated values and they are not of the same accuracy as the ones from the adjusted Breit-Pauli calculations of Froese Fischer and Tachiev [21]. As expected, the agreement between the present values and the values from Breit-Pauli calculations becomes better when $Z$ increases.

\section{Summary and conclusion}

We have shown that fully relativistic multiconfiguration calculations have the capability to provide accurate hyperfine and isotope shift parameters for light atoms. The relativistic approach yields less accurate rates of intercombination transitions in light ions near the neutral end, but the accuracy improves rapidly with nuclear charge. Already for $\mathrm{Ne} \mathrm{V}$ the relativistic transition rates compare very well with values from recent Breit-Pauli calculations. In contrast to the BreitPauli calculations, the relativistic computational scheme can be applied with good accuracy up to the high- $Z$ end of the iso-electronic sequence [48]. An optimal tool for calculations of atomic properties along an isoelectronic sequence would be a combination of a Breit-Pauli method and a fully relativistic Dirac-Hartree-Fock method. The former should be applied near the neutral end of the sequence, where it has the advantage in more accurate treatment of electron correlation effects. At some point along the sequence both methods yield results of comparable accuracy, and the Breit-Pauli approach begins to break down soon after. The Dirac-Hartree-Fock method would take over from there. To achieve a better description of energy separations it is desirable to develop systematic and automated procedures for selecting the multireference sets. Larger multireference sets make the configuration expansions grow very rapidly and the calculations would have to be run on parallel systems using the MPI version of the GRASP2K code [47]. Also, it would be of great interest to develop a fine-tuning scheme in $j j$-coupling. This is, however, not a straightforward task, since such a scheme-in contrast to the one in the Breit-Pauli approximation-involves also a modification of off-diagonal matrix elements in the Hamiltonian.

\section{Acknowledgment}

Financial support by the Swedish Research Council is gratefully acknowledged.

\section{References}

[1] Lennon D L, Dufton P L, Hibbert A and Kingston A E 1985 Astrophys. J. 294200

[2] Keenan F P, Dufton P L, Feibelman W A, Bell K L, Hibbert A and Stafford R P 1994 Astrophys. J. 423882

[3] Brage T, Judge P G and Brekke P 1996 Astrophys. J. 4641030

[4] Eissner W, Jones M and Nussbaumer H 1974 Comput. Phys. Commun. 8270

[5] Hibbert A 1975 Comput. Phys. Commun. 9141

[6] Froese Fischer C, Tachiev G, Gaigalas G and Godefroid M R 2007 Comput. Phys. Commun. 176559

[7] Froese Fischer C, Brage T and Jönsson P 1997 Computational Atomic Structure-An MCHF Approach (Bristol: Institute of Physics Publishing)

[8] Glass R and Hibbert A 1978 Comput. Phys. Commun. 1619
[9] Hummer D G, Berrington K A, Eissner W, Pradhan A K, Saraph H E and Tully J A 1993 Astron. Astrophys. 279298

[10] Hibbert A 1996 Phys. Scr. T 65104

[11] Pyykkö P 1988 Chem. Rev. 88563

[12] Jönsson P, He X, Froese Fischer C and Grant I P 2007 Comput. Phys. Commun. 177597

[13] Indelicato P and Desclaux J-P http://dirac.spectro.jussieu.fr/mcdf

[14] Olsen J, Godefroid M R, Jönsson P, Malmqvist P A and Froese Fischer C 1995 Phys. Rev. E 524499

[15] Jönsson P, Parpia F A and Froese Fischer C 1996 Comput. Phys. Commun. 96301

[16] Jönsson P and Froese Fischer C 1997 Comput. Phys. Commun. 94249

[17] Andersson M and Jönsson P 2008 Comput. Phys. Commun. 178156

[18] Feibelman W A, Hyung S and Aller L H 1996 MNRAS 278625

[19] Kurucz R L 1993 Phys. Scr. T 11047

[20] Tachiev G and Froese Fischer C 2001 Can. J. Phys. 79955

[21] Froese Fischer C and Tachiev G 2004 At. Data Nucl. Data Tables 871

[22] Aggarwal K M, Hibbert A and Keenan F P 1997 Astrophys. J. Suppl. Ser. 108393

[23] Aggarwal K M 1998 Astrophys. J. Suppl. Ser. 118589

[24] Aggarwal K M, Keenan F P and Msezane A Z 2001 Astrophys. J. Suppl. Ser. 136763

[25] Bhatia A K and Doschek G A 1993 At. Data Nucl. Data Tables 55315

[26] Fawcett B C 1987 At. Data Nucl. Data Tables 37367

[27] Grant I P 2007 Relativistic Quantum Theory of Atoms and Molecules (New York: Springer)

[28] McKenzie B J, Grant I P and Norrington P H 1980 Comput. Phys. Commun. 21233

[29] Johnson W R and Soff G 1985 At. Data Nucl. Data Tables 33405

[30] Welton T A 1948 Phys. Rev. 741157

[31] Dyall K G, Grant I P, Johnson C T, Parpia F A and Plummer E P 1989 Comput. Phys. Commun. 55425

[32] Jönsson P and Froese Fischer C 1994 Phys. Rev. A 50 3080

[33] Andersson M, Jönsson P and Sabel H 2006 J. Phys. B: At. Mol. Phys. 394239

[34] Stone N J 2005 At. Data Nucl. Data Tables 9075

[35] Froese Fischer C and Jönsson P 2001 J. Mol. Struct. 53755

[36] Grant I P 1974 J. Phys. B: At. Mol. Phys. 71458

[37] Malmqvist P Å 1986 Int. J. Quantum Chem. 30479

[38] Jönsson P and Froese Fischer C 1993 Phys. Rev. A 484113

[39] Clark C W 1984 Astrophys. J. 285322

[40] Ralchenko Y, Kramida A E and Reader J 2008 (NIST ASD Team). NIST Atomic Spectra Database (v 3.1.5) [online]. Available: http://physics.nist.gov/asd3 [2008, June 26] National Institute of Standards and Technology, Gaithersburg, MD

[41] http://physics.nist.gov/PhysRefData/ASD/index.html

[42] Ynnerman A and Froese Fischer C 1995 Phys. Rev. A 512020

[43] Chen M H, Cheng K T and Johnson W R 2001 Phys. Rev. A 64042507

[44] Cheng K T, Kim Y K and Desclaux J P 1979 At. Data Nucl. Data Tables 24111

[45] Brage T, Hibbert A and Leckrone D S 1997 Astrophys. J. 478423

[46] Fleming J and Brage T 1997 J. Phys. B: At. Mol. Phys. 301385

[47] Bieroń J, Froese Fischer C, Indelicato P, Jönsson P and Pyykkö P 2009 Phys. Rev. A 79052502

[48] Boucard S and Indelicato P 2000 Eur. Phys. J. D 8 59-73

[49] Calamai A G and Johnson C E 1991 Phys. Rev. A 44218 
[50] Träbert E, Wolf A, Pinnington E H, Linkemann J, Knystautas E J, Curtis A, Bhattacharya N and Berry H G 1998 Phys. Rev. A 584449

[51] Doschek G A, Feldman U and Cohen L 1977 Astrophys. J. Suppl. 33101

[52] Bridges J M, Wiese W L and Griesmann U 1996 Goddard High Resolution Spectrograph Science Symposium Greenbelt, MD, p 40

[53] Curry J, Gibson N D and Lawler J E 1997 Astron. Astrophys. 3211021
[54] Musielok J, Bridges J M, Djurović S and Wiese W L 1996 Phys. Rev. A 533122

[55] Johnson B C, Smith P L and Knight R D 1984 Astrophys. J. 281477

[56] Träbert E, Calamai A G, Gillaspy J D, Gwinner G, Tordoir X and Wolf A 2000 Phys. Rev. A 62022507

[57] Doschek G A 1996 private communication quoted in [46]

[58] Harper G M 1996 private communication quoted in [46] 